\title{
ON DIMENSION OF SUPPORT FOR STOCHASTIC PROCESSES WITH INDEPENDENT INCREMENTS $\left({ }^{1,2}\right)$
}

\author{
BY \\ HOWARD H. STRATTON, JR.
}

Introduction. Herman Rubin [5] showed that if $v(t)$ is a real valued function which is monotone nondecreasing on the interval $(0, \alpha)$, where $\alpha$ is possibly $+\infty$, and if $0 \leqq v(t) \leqq 1$, then there exists a "pure" stochastic process $\left\{X_{t}: t \in[0, \infty)\right\}$ (i.e. for $t \in(0, \infty)$ the distribution function of $X_{t}$, denoted by $F_{t}$, is either absolutely continuous or continuous singular) with stationary independent increments such that if $t<\alpha$, then $F_{t}$ is continuous singular with the dimension of its support, denoted by $\operatorname{dim} \operatorname{supp} F_{t}$, being $v(t)$; and if $t \leqq \alpha$, then $F_{t}$ is absolutely continuous. This can be interpreted as saying that for a "pure" process with stationary independent increments, the only general properties the dimension of the support of $F_{t}$ has to have as a function of $t$, are the obvious ones of being monotone nondecreasing and being bounded between 0 and 1 .

One is now led to remove the restriction that the process be "pure," to isolate one's attention to the continuous singular component of $F_{t}$, denoted by $\left(F_{t}\right)$ c.s., and to ask if a similar result to that of Rubin's holds for $\operatorname{dim} \operatorname{supp}\left(F_{t}\right)$ c.s. The answer is yes. In $\S 1$ of this paper, it is shown that if $\left\{X_{t}: t \in[0, \infty)\right\}$ is a stochastic process with independent increments then

$$
\operatorname{dim} \operatorname{supp}\left(F_{t}\right) \text { c.s. }=\liminf _{\tau \rightarrow t} \operatorname{dim} \operatorname{supp}\left(F_{\tau}\right) \text { c.s. }
$$

for all $t \in(0, \infty)$ except at possibly a countable number of points. Conversely, in $\S 2$ it is shown that if $f$ maps $(0, \infty)$ into $[0,1]$ and is a lower semicontinuous function at all but possibly a countable number of points, then there exists a stochastic process $\left\{X_{t}: t \in[0, \infty)\right\}$ with stationary independent increments such that $\operatorname{dim} \operatorname{supp}\left(F_{t}\right)$ c.s. $=f(t)$.

These two results are obtained while investigating the relationship between the total variation of $\left(F_{t}\right)$ c.s., denoted by T.V. $\left(F_{t}\right)$ c.s., and $\operatorname{dim} \operatorname{supp}\left(F_{t}\right)$ c.s. for a process with independent increment.

At this point I wish to express my gratitude to Professor Howard G. Tucker for

Received by the editors May 23, 1966.

$\left.{ }^{1}\right)$ This paper is a dissertation submitted in partial satisfaction of the requirements of a graduate program in mathematics for the degree of Doctor of Philosophy at the University of California, Riverside.

(2) This research was supported in part by the Air Force Office of Scientific Research, Grant No. AF-AFOSR 851-65 and by the U.S. Army Research Office, Grant No. DA-ARO(D)31-124-G383. 
directing my attention to this problem, his patience in listening to my ideas, and his persistence in encouraging my endeavors.

I wish also to express my gratitude to Mrs. Jane Scully who skillfully typed this paper.

I. In this section we establish the following. Let $\left\{X_{t}: t \in[0, \infty)\right\}$ be a stochastic process with independent increments. A theorem is proved concerning how T.V. $\left(F_{t}\right)$ c.s., having the analytic property of being continuous from the right at $t_{0}$, affects the function $v(t) \doteq \operatorname{dim} \operatorname{supp}\left(F_{t}\right)$ c.s. at $t_{0}$. Furthermore it is proved that $\operatorname{dim} \operatorname{supp}\left(F_{t}\right)$ c.s. $=\lim \inf _{\tau \rightarrow t} \operatorname{dim} \operatorname{supp}\left(F_{\tau}\right)$ c.s. except at possibly a countable number of points. Secondly for processes with stationary independent increments a relation along the lines of the obvious one that if T.V. $\left(F_{t}\right)$ c.s. $\cdot$ T.V. $\left(F_{d}\right)$ c.s. $=$ T.V. $\left(F_{t+d}\right)$ c.s. then $\operatorname{dim} \operatorname{supp}\left(F_{t+d}\right)$ c.s. $\geqq \max \left(\operatorname{dim} \operatorname{supp}\left(F_{t}\right)\right.$ c.s., $\operatorname{dim} \operatorname{supp}\left(F_{d}\right)$ c.s. $)$ is proved.

For completeness we give the following definitions:

Notation. Let $A$ be a Lebesgue measurable subset of the real line; then $|A|$ shall denote the Lebesgue measure of $A$.

Definition. Let $A$ be a subset of the real line, then the Hausdorff dimension of $A$, $\operatorname{dim}(A)$ is defined as follows:

Let

$$
\mathscr{I}_{\xi}(A) \doteq\left\{\left\{I_{i}\right\}: I_{i} \text { is an open interval, } \bigcup I_{i} \supset A \text {, and }\left|I_{i}\right|<\xi\right\},
$$

let $u_{\xi}^{\alpha}(A)=\inf \left\{\sum_{i=1}^{\infty}\left|I_{i}\right|^{\alpha}:\left\{I_{i}\right\}_{i=1}^{\infty} \in \mathscr{I}_{\xi}(A)\right\}$, and define

$$
\operatorname{dim}(A)=\inf \left\{\alpha: \lim _{\xi \rightarrow 0} u_{\xi}^{\alpha}(A)=0\right\} .
$$

Notation. (The word "Borels" will always denote in the sequel the sigma algebra of Borel sets.) Let $G$ be a nondecreasing function, and let $A \in$ Borels; then $G(A)$ will denote the value that the measure induced by $G$ on the Borels assigns to $A$. Also let $(G)$ c.s., $(G)$ a.c. and $(G)$ d. denote the continuous singular, absolutely continuous, and discrete components of $G$ respectively.

Definition. Let $G$ be a nondecreasing function; the dimension of the support of $G, \operatorname{dim} \operatorname{supp} G$, is defined as

$$
\operatorname{dim} \operatorname{supp} G=\inf \{\operatorname{dim}(A): A \in \text { Borels, } G(A)=\text { T.V.(G) }\} .
$$

Notation. Let $t \in[0, \infty)$; then let

$$
[t]=\max \left\{n \in I^{+} \cup\{0\}: n \leqq t\right\} .
$$

We shall assume the following six facts which can be proved by straightforward elementary techniques.

A.1. If $\left\{A_{i}\right\}$ is a countable collection of sets, then

$$
\operatorname{dim}\left(\bigcup_{i} A_{i}\right)=\max _{i} \operatorname{dim}\left(A_{i}\right) .
$$

Also if $A$ is a set and $x \in(-\infty, \infty)$, then

$$
\operatorname{dim}(A)=\operatorname{dim}(A+x) .
$$


A.2. Let $\left\{G_{t}(x): t \in\right.$ Reals $\}$ be a collection of distribution functions in $x$ such that for fixed $x, G_{t}(x)$ is a Borel measurable function in $t$. Let $m$ be a bounded positive measure on the Borels and let $V(x)=\int_{-\infty}^{\infty} G_{t}(x) d m(t)$. If $A$ is a Borel set, then $G_{t}(A)$ is a Borel measurable function in $t$ and

$$
V(A)=\int_{-\infty}^{\infty} G_{t}(A) d m(t)
$$

Also if $V(A)=$ T.V. $(m)$, then $G(A)=1$ a.e. $[m]$.

A.3. If $G$ and $F$ are nondecreasing functions, then $\operatorname{dim} \operatorname{supp} G * F \geqq \operatorname{dim} \operatorname{supp} F$.

A.4. If $G$ and $F$ are nondecreasing functions, and $G$ is discrete, then

$$
\operatorname{dim} \operatorname{supp} G * F=\operatorname{dim} \operatorname{supp} F \text {. }
$$

A.5. Let $\left\{G_{n}\right\}$ be a collection of nondecreasing functions such that $G(x)=$ $\sum_{n} G_{n}(x)<\infty$ for every $x$. If $V$ is a nondecreasing function then $G * V=\sum_{n} G_{n} * V$ and $\operatorname{dim} \operatorname{supp} G=\max _{n} \operatorname{dim} \operatorname{supp} G_{n}$.

A.6. Let $\left\{F_{n}\right\}$ be a sequence of real valued bounded nondecreasing functions which are continuous from the right at every point $x \in(-\infty, \infty)$. Then $F_{n} \stackrel{(c)}{\rightarrow} F_{0}$ if and only if for every Borel set $A$ such that $F_{0}$ (boundary $\left.(A)\right)=0$, then $F_{n}(A) \vec{n}$ $F(A)\left(F_{n} \stackrel{(c)}{\rightarrow} F_{0}\right.$ means $F_{n}(x) \rightarrow F_{0}(x)$ for all continuity points of $\left.F_{0}\right)$.

LEMMA 1.1. Let $G(x)$ be a nondecreasing function in $x$, and assume $\operatorname{dim} \operatorname{supp} G>\alpha$. Then there exists $a \delta_{\alpha}>0$ such that if $A$ is a Borel set and $\operatorname{dim}(A) \leqq \alpha$, then $G(A)<$ T.V. $G-\delta_{\alpha}$.

Proof. Suppose not. Then clearly there exists a countable sequence $\left\{A_{i}\right\}$ of Borel sets such that $\operatorname{dim}\left(A_{i}\right) \leqq \alpha$ for each $i$, and $G\left(A_{i}\right) \rightarrow \vec{i}$ T.V.(G). By the continuity of measures we see that $G\left(\bigcup_{i} A_{i}\right)=$ T.V.(G). But by A.1. we see that $\operatorname{dim}\left(\bigcup A_{i}\right) \leqq \alpha$ which, by the definition of $\operatorname{dim} \operatorname{supp} G$, implies that $\operatorname{dim} \operatorname{supp} G \leqq \alpha$, which is a contradiction.

For the rest of this section we adopt the following notation:

Let $\left\{X_{t}: t \in[0, \infty)\right\}$ be a stochastic process; then

$$
m(t)=\text { T.V. }\left(F_{t}\right) \text { c.s., } \quad m_{a}(t)=\text { T.V. }\left(F_{t}\right) \text { a.c., } \quad m_{d}(t)=\text { T.V. }\left(F_{t}\right) \mathrm{d} .
$$

and if $s>t$

$$
\begin{gathered}
F_{t, s}=F_{X_{s}-X_{t}}, \quad m(t, s)=\text { T.V. }\left(F_{t, s}\right) \text { c.s., } \quad m_{a}(t, s)=\text { T.V. }\left(F_{t, s}\right) \text { a.c., } \\
m_{d}(t, s)=\text { T.V. }\left(F_{t, s}\right) \text { d. }, \quad v(t)=\operatorname{dim} \operatorname{supp}\left(F_{t}\right) \text { c.s. }
\end{gathered}
$$

Let $\left\{X_{t}: t \in[0, \infty)\right\}$ be a stochastic process with independent increments. So if $s, t \in(0, \varepsilon)$ and $s>t$, then

$$
F_{s}=\left(\left(F_{t}\right) \text { c.s. }+\left(F_{t}\right) \text { a.c. }+\left(F_{t}\right) \text { d. }\right) *\left(\left(F_{t, s}\right) \text { c.s. }+\left(F_{t, s}\right) \text { a.c. }+\left(F_{t, s}\right) \text { d. }\right) .
$$


From this equality it is easily seen that the following key relations hold.

R.1. $\left(F_{s}\right)$ a.c. $=\left(F_{t}\right)$ a.c. $*\left(F_{t, s}\right)+\left(\left(F_{t}\right)\right.$ c.s. $+\left(F_{t}\right)$ d. $) *\left(F_{t, s}\right)$ a.c. $+\left(\left(F_{t}\right)\right.$ c.s. $*\left(F_{t, s}\right)$ c.s. $)$ a.c.,

R.2. $\left(F_{s}\right)$ c.s. $=\left(F_{t}\right)$ c.s. $*\left(F_{t, s}\right)$ d. $+\left(F_{t, s}\right)$ c.s. $*\left(\mathrm{~F}_{t}\right)$ d. $+\left(\left(F_{t}\right)\right.$ c.s. $*\left(F_{t, s}\right)$ c.s. $)$ c.s.,

R.3. $\left(F_{s}\right) \mathrm{d} .=\left(F_{t}\right) \mathrm{d} . *\left(F_{t, s}\right) \mathrm{d}$.

THEOREM. Let $\left\{X_{t}: t \in[0, \infty)\right\}$ be a stochastic process with independent increments. Let $d_{0}=1$.u.b. $\left\{x \in[0, \infty): m_{d}(x) \neq 0\right\}$. Under these assumptions the function $v(t)$ has the following properties:

1. $v(t)$ is monotone increasing on $\left(0, d_{0}\right)$;

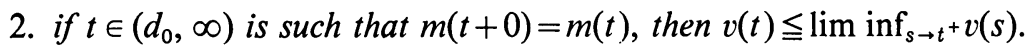

3. $v(t)=\lim _{\inf _{s \rightarrow t}} v(s)$ for all but a countable number of points.

Proof. In order to establish property 1 , we first show that if $t \in\left(0, d_{0}\right)$ then $m_{d}(t) \neq 0$. So suppose there exists $t \in\left(0, d_{0}\right)$ such that $m_{d}(t)=0$. Let $s \in\left(t, d_{0}\right]$. Then by R. 3 we see that $m_{d}(s)=0$. This contradicts the definition of $d_{0}$ and establishes the fact that if $t \in\left(0, d_{0}\right)$ then $m_{d}(t) \neq 0$.

In order to complete the proof of property 1 , we let $s_{1}, s_{2} \in\left(0, d_{0}\right)$ with $s_{1}>s_{2}$. By the definition of $d_{0}$ we see that $m_{d}\left(s_{2}\right) \neq 0$ and therefore by R.3 we see that $m_{d}\left(s_{2}, s_{1}\right) \neq 0$.

Now recalling R.2, A.4 and A.5 we see that $v\left(s_{2}\right)>v\left(s_{1}\right)$ which shows property 1 holds.

To establish property 2 we let $t \in\left(d_{0}, \infty\right)$ such that $m(t+0)=m(t)$. By the definition of $d_{0}$ we see that $m_{d}(t)=0$. So referring to R.2 we see that for $A \in$ Borels

$$
\begin{aligned}
\left(F_{s}\right) \text { c.s. }(A) & \leqq\left(F_{t}\right) \text { c.s. } *\left(F_{t, s}\right) \text { d. }(A)+\left(F_{t}\right) \text { c.s. } *\left(F_{t, s}\right) \text { c.s. }(A) \\
& \leqq \int_{-\infty}^{\infty}\left(F_{t}\right) \text { c.s. }(A-x) d\left(\left(F_{t, s}\right) \text { c.s. }+\left(F_{t, s}\right) \text { d. }\right)(x) .
\end{aligned}
$$

The conclusion of the theorem not holding at $t$ means that there exists a real number $\beta$ and a sequence of real numbers $\left\{s_{j}\right\}$ such that for every $j \in I^{+}$we have $v\left(s_{j}\right)<\beta<v(t)$ and $s_{j} \downarrow t$. For $j \in I^{+}$, we see that by the definition of dim supp there exists a set $A_{j}$ such that

$$
\left(F_{s_{j}}\right) \text { c.s. }\left(A_{j}\right)=m\left(s_{j}\right) \text { and } \operatorname{dim}\left(A_{j}\right)<\beta .
$$

Let

$$
A=\bigcup_{j=1}^{\infty} A_{j}
$$

By A.1. $\operatorname{dim}(A) \leqq \beta$, and so by Lemma 1.1 we see that, for every $x \in(-\infty, \infty)$,

$$
\left(F_{t}\right) \text { c.s. }(A-x)<m(t)-\delta_{\beta} \text { for some } \delta_{\beta}>0 \text {. }
$$

Now by (1.2) $\left(F_{s_{j}}\right)$ c.s. $(A)=m\left(s_{j}\right)$ for all $j \in I^{+}$and therefore by (1.1), (1.3) and the fact that $m(t, s)+m_{d}(t, s) \leqq 1$, we see that

$$
m\left(s_{j}\right) \leqq m(t)-\delta_{\beta} .
$$


Letting $j \rightarrow \infty$ on both sides of the inequality in (1.4) we see that, by the fact that $m(t)=m(t+0), m(t) \leqq m(t)-\delta_{\beta}$. But $\delta_{\beta}>0$, which gives a contradiction and the completion of the proof of 2 .

Now to prove 3 of this theorem we note the following. R.3 and the fact that $m_{d}(t, s) \leqq 1$ implies $m_{d}(t)$ is nonincreasing for increasing $t$, and therefore $m_{d}(t)$ is continuous from the right at all except possible countably many points $t$. R.1 clearly implies that $m_{a}(t)$ is a monotone increasing function and therefore is continuous from the right for all except possibly countably many points $t$. But $1-m_{a}(t)-m_{d}(t)=m(t)$ and therefore $m$ is continuous from the right for all except possibly countably many points $t$. So by 2 of this theorem

$$
v(t) \leqq \liminf _{s \rightarrow t^{+}} v(s)
$$

holds for all except possibly countably many points $t$.

Saks ([6], page 261) shows that any real valued function $F$ has the property that the set $\left\{x: \lim \inf _{t \rightarrow x} F(t)<\lim _{t \rightarrow x^{+}} F(t)\right\}$ is at most denumerable. Therefore we see that $v(t) \leqq \lim _{\inf } \rightarrow t(s)$ for all but a countable number of points $t$. Showing that this in turn implies that $v(t)=\lim _{s \rightarrow t} v(s)$ at all but a countable number of points $t$ is a standard argument and is therefore omitted. This then completes the proof of the theorem.

II. In this section we establish the following theorem:

THEOREM. Let a function $m(t)$ which maps, $[0, \infty)$ into $[0,1]$, be such that

1. $m(t+d) \geqq m(t) m(d)$ for $t, d \in[0, \infty)$,

2. there exists $t^{*} \in(0, \infty)$ such that $m\left(t^{*}\right)=0$ or 1 , and

3. on the interval $\left\{x \in(0, \infty): m(x) \neq 0\right.$ and $\left.\ln m(t) / t \neq m^{\prime}(0)\right\}, \ln m(t) / t$ is a strictly increasing function.

Let a function $v(t)$ which maps $(0, \infty)$ into $[0,1]$ be such that

1. $v(t)=0$ on the interval $B=\{t \in(0, \infty): m(t)=0\}$,

2. $v(t)$ is monotone increasing on the interval $A=\{t \in(0, \infty): m(t) / t=m(0)\}$, and

3. $v(t) \leqq \lim _{s \rightarrow t^{+}} v(s)$ if $t \in(0, \infty)-B$ and $m(t)=m(t+0)$.

Then there exists a stochastic process, $\left\{X_{t}: t \in[0, \infty)\right\}$, with stationary independent increments such that for $t \in(0, \infty):$ T.V. $\left(F_{t}\right)$ c.s. $=m(t)$ and $\operatorname{dim} \operatorname{supp}\left(F_{t}\right)$ c.s. $=v(t)$.

As a corollary to the above result we find that if $\alpha$ is a real extended number and if $f$ is a function on $(0, \alpha)$ (or $(0, \alpha])$ which is lower semicontinuous at all but a countable number of points and is bounded between 0 and 1 , then there exists a stochastic process $\left\{X_{t}: t \in[0, \infty)\right\}$, with stationary independent increments such that $f(t)=\operatorname{dim} \operatorname{supp}\left(F_{t}\right)$ c.s. for $t \in(0, \alpha)$ (or $\left.(0, \alpha]\right)$ and $F_{t}$ is absolutely continuous for $t \geqq \alpha($ or $t>\alpha)$.

In the first two of the following eight lemmas, let $\left\{M_{n}\right\}$ be a sequence of bounded negative functions such that $M_{n}(s)$ increases with increasing $x, \sum_{n} M_{n}(x)=$ $M(x)>-\infty$ for every $x \in(0, \infty)$, and $\int_{0}^{1} x d M(x)<\infty$. Let $\lambda_{n}=$ T.V. $\left(M_{n}\right)$ and let 
$F_{n, m}(x)$ be the distribution function corresponding to the characteristic function

$$
\exp \left(\int_{0^{+}}^{\infty}(\exp (i \mu x)-1) d\left\{\sum_{j=n+1}^{m} M_{j}(x)\right\}\right)
$$

where $m>n, n \in\{0\} \cup I^{+}$, and $m \in I^{+} \cup\{+\infty\}$.

LEMMA 2.1. Let $k \in I^{+}$; then there exists a nondecreasing function $F_{k}^{\prime}(x)$ such that if $n>k$

$$
\begin{aligned}
& F_{k}^{n}(x) \doteq\left\{\exp \left(-\sum_{i=0}^{n} \lambda_{i}\right)\right\} \sum_{i=0}^{\infty}\left(\sum_{j=k}^{n} M_{j}\right)^{* i}(x) / i ! \stackrel{(c)}{\longrightarrow} F_{k}^{\prime}(x), \\
& \text { T.V. }\left(F_{k}^{\prime}\right)=\exp \left(-\sum_{i=1}^{k-1} \lambda_{i}\right), \text { and } F_{k}^{n} * F_{n, \infty}(x)=F_{k}^{\prime}(x) .
\end{aligned}
$$

Proof. Let us denote the characteristic function of $F$ by ch.fcn. $(F)$. Clearly

$$
\text { ch.fcn. }\left(F_{k}^{n}\right)=\exp \left(-\sum_{i=0}^{n} \lambda_{i}\right) \exp \left(\int_{0^{+}}^{\infty}(\exp (i u x)) d\left(\sum_{j=k}^{n} M_{j}(x)\right)\right) .
$$

But T.V. $\left(\sum_{j=k}^{n} M_{j}\right)=\sum_{j=k}^{n} \lambda_{j}$ which implies

$$
\text { ch.fcn. }\left(F_{k}^{n}\right)=\exp \left(-\sum_{i=1}^{k-1} \lambda_{i}\right) \operatorname{ch} . f c n .\left(F_{k-1, n}\right) \text {. }
$$

By definition $\sum_{j=k}^{n} M_{j}(x) \underset{n}{\rightarrow} \sum_{j=k}^{\infty} M_{j}(x)$ and therefore by [3]

So let

$$
\text { ch.fcn. }\left(F_{k}^{n}\right) \underset{n}{\longrightarrow} \operatorname{ch} . \text { fcn. }\left(F_{k-1, \infty}\right) \exp \left(-\sum_{j=0}^{k-1} \lambda_{j}\right) \text {. }
$$

Therefore

$$
F_{k}^{\prime}(x)=\left\{\exp \left(-\sum_{i=0}^{k-1} \lambda_{i}\right)\right\} F_{k-1, \infty}(x)
$$

$$
F_{k}^{n}(x) \stackrel{(c)}{\longrightarrow} F_{k}^{\prime}(x) \text {. }
$$

But T.V. $\left(F_{k}^{n}\right)=\exp \left(-\sum_{i=0}^{k-1} \lambda_{i}\right)$ and so by the definition of complete convergence T.V. $\left(F_{k}^{\prime}\right)=\exp \left(-\sum_{i=0}^{k-1} \lambda_{i}\right)$.

The form of the characteristic function of $F_{k-1, \infty}$ clearly implies that for $m>k$, $F_{k-1, \infty}=F_{k-1, m} * F_{m, \infty}$. Now multiplying both sides of this equation by $\exp \left(-\sum_{j=0}^{k-1} \lambda_{j}\right)$ we get the last statement of the lemma and the completion of the proof.

Lemma 2.2. Let $V$ be a continuous distribution function and $k \in I^{+}$. For each $n \in I^{+}$, let $\mathscr{I}_{n}^{\prime}=\left\{I_{j, n}^{\prime}: j \in I^{+}\right\}$and $\mathscr{I}_{n}=\left\{I_{j, n}: j \in I^{+}\right\}$be two collections of open intervals where the collection of endpoints from intervals in $\mathscr{I}_{n}$ has no limit points, and $V\left[\bigcup_{n=N}^{\infty} \bigcup_{j=1}^{\infty} I_{j, n}^{\prime}\right]=1$ for every $N \in I^{+}$. If

1. there exists an increasing sequence $\left\{j_{n}\right\}_{n=1}^{\infty} \subset I^{+}$such that $\sum_{v=1}^{\infty}\left(\sum_{n=1}^{v} \lambda\right)^{s_{v}} / j_{v}$ ! $<\infty$, and if 
2. there exists a sequence of real numbers $\left\{\xi_{n}\right\}$ where $\xi_{n} \downarrow 0$ and an unbounded sequence $\left\{n_{i}\right\} \subset I^{+}$such that for $n, N, m \in I^{+}$and $m \geqq N$ :

$$
\underset{s=k}{*} M_{s}^{* e_{s}} * V\left[\bigcup_{n=N}^{m} \bigcup_{j=1}^{\infty} I_{j, n}\right] \geqq\left(1-\xi_{N}\right) \prod_{s=k}^{n} \lambda_{s}^{e_{s}} V\left[\bigcup_{n=N}^{m} \bigcup_{j=1}^{\infty} I_{j, n}^{\prime}\right]
$$

whenever $\sum_{s=k}^{l} e_{s}<j_{l}$ for each $l=n_{N}, \ldots, n$, then, for every $r \in I^{+}$,

$$
F_{k}^{\prime} * V\left[\bigcup_{n=r}^{\infty} \bigcup_{j=1}^{\infty} I_{j, n}\right]=\exp \left(-\sum_{i=1}^{k-1} \lambda_{i}\right)=\text { T.V. }\left[F_{k}^{\prime} * V\right] .
$$

Proof. Let $U_{N, m}=\bigcup_{n=N}^{m} \bigcup_{j=1}^{\infty} I_{n, j}$, and let $U_{N, m}^{\prime}=\bigcup_{n=N}^{m} \bigcup_{j=1}^{\infty} I_{n, j}^{\prime}$ where $m \geqq N$ and $m$ is possibly $+\infty$. Now in order to prove this lemma, it is clearly sufficient to show that for $\varepsilon>0$, there exists $N(\varepsilon) \in I^{+}$such that $N \geqq N(\varepsilon)$ implies

1. $F_{k}^{\prime} * V\left(U_{N, \infty}\right) \geqq \exp \left(-\sum_{i=1}^{k-1} \lambda_{i}\right)-\varepsilon$.

By hypothesis $V$ is a continuous distribution function and therefore $F_{k}^{\prime} * V$ is a continuous distribution function. So if $m \neq+\infty$, then

$$
F_{k}^{\prime} * V\left(\text { Boundary }\left(U_{N, m}\right)\right)=F_{k}^{\prime} * V(\text { countable set })=0
$$

and therefore by A.6

$$
F_{n}^{k} * V\left(U_{N, m}\right) \underset{k}{\longrightarrow} F_{k}^{\prime} * V\left(U_{N, m}\right) .
$$

But from this and the hypothesis that $V\left(U_{N, \infty}^{\prime}\right)=1$ for every $N \in I^{+}$, we see that in order to prove 1 it is sufficient to verify the following:

If $\varepsilon>0$, then there exists $N(\varepsilon)$ such that $N \geqq N(\varepsilon)$ and $n \geqq \max \left(n_{N}, k\right)$ imply

$$
F_{n}^{k} * V\left(U_{N, m}\right) \geqq \exp \left(-\sum_{i=1}^{k-1} \lambda_{l}\right) V\left(U_{N, m}^{\prime}\right)-\varepsilon .
$$

So let $\varepsilon>0$. Since $\left\{n_{i}\right\}$ is an unbounded sequence of integers we see from hypothesis 1 that there exists $N^{\prime} \in I^{+}$such that if $N \geqq N^{\prime}$ then

$$
\exp \left(\sum_{s=1}^{k-1} \lambda_{s}\right) \sum_{v=n_{N}}^{\infty}\left(\sum_{n=1}^{v} \lambda_{n}\right)^{j_{v}} / j_{v} !<\varepsilon / 2 .
$$

Since $\varepsilon_{N} \downarrow 0$ there exists $N_{1} \in I^{+}$such that if $N \geqq N_{1}$ then

$$
\exp \left(-\sum_{i=1}^{k-1} \lambda_{i}\right) \varepsilon_{N}<\varepsilon / 2
$$

Let $N(\varepsilon)=\max \left(N_{1}, N^{\prime}\right)$ and let $N \geqq N_{\varepsilon}$. Finally let $n \geqq \max \left(n_{N}, k\right)$.

Let

$$
\begin{aligned}
R_{i} & =\left\{e \in{\underset{s}{s=k}}_{x}^{n}\{1, \ldots, i\}_{s}: \sum_{s=k}^{n} e_{s}=i\right\}, \\
R_{i, r}^{\prime} & =\left\{e \in R_{i}: \text { for each } l=n_{N}, \ldots, r ; \sum_{s=k}^{l} e_{s}<j_{l}\right\}, \\
R_{i, r} & =\left\{e \in R_{i}: \text { for some } l, n_{N} \leqq l \leqq r, \sum_{s=k}^{l} e_{s} \geqq j_{l}\right\} .
\end{aligned}
$$


Now clearly $R_{i, r} \cap R_{i, r}^{\prime}=\varnothing$ and $R_{i, r} \cup R_{i, r}^{\prime}=R_{i}$. So

$$
\begin{aligned}
F_{n}^{k} * V\left(U_{N, m}\right)= & \exp \left(-\sum_{i=1}^{n} \lambda_{i}\right)\left(\sum_{i=0}^{\infty}\left(\sum_{j=k}^{n} M_{j}\right)^{* i} * V / i !\right)\left(U_{N, m}\right) \\
\geqq & \exp \left(-\sum_{i=1}^{n} \lambda_{i}\right)\left(\sum_{i=1}^{j_{n}-1}\left(\sum_{j=k}^{n} M_{j}\right)^{* i} * V / i !\right) \\
& \quad+\sum_{r=n_{N}}^{n-1} \sum_{i=j_{r}}^{j_{r}+1-1}(V / i !) * \sum_{e \in R_{i, r}^{i}} \underset{s=k}{*} M_{s}^{* e_{s}}\left(\begin{array}{c}
1-\sum_{t=k}^{s-1} e_{t} \\
e_{s}
\end{array}\right)\left(U_{N, m}\right) \\
\geqq & \left(1-\varepsilon_{N}\right) V\left(U_{N, m}^{\prime}\right) \\
& \times\left\{\exp \left(-\sum_{i=k}^{n} \lambda_{i}\right)-\exp \left(-\sum_{i=1}^{n} \lambda_{i}\right)\right. \\
& \times\left(\sum_{i=j_{n}}^{\infty}\left(\sum_{s=k}^{n} \lambda_{s}\right)^{i} / i !-\sum_{r=n_{N}}^{n-1} \sum_{i=j_{r}}^{j_{r}+1-1}(1 / i !) \sum_{e \in R_{i, r}} \prod_{s=k}^{n} \lambda_{s}^{e_{s}}\left(\begin{array}{c}
1-\sum_{t=k}^{s-1} e_{t} \\
e_{s}
\end{array}\right)\right\} .
\end{aligned}
$$

First let us note that by Taylor's theorem

$$
\sum_{i=j_{n}}^{\infty}\left(\sum_{s=k}^{n} \lambda_{s}\right)^{i} / i ! \leqq \exp \left(\sum_{s=k}^{n} \lambda_{s}\right)\left(\sum_{s=k}^{n} \lambda_{s}\right)^{j_{n}} / j_{n} !
$$

But recall that $n \geqq n_{N}$, and therefore

$$
\exp \left(-\sum_{s=1}^{n} \lambda_{s}\right) \sum_{i=j_{n}}^{\infty}\left(\sum_{s=k}^{n} \lambda_{s}\right)^{i} / i !<\varepsilon / 2
$$

Now let $n-1 \geqq r \geqq l$ and $P \in I^{+}$be such that $j_{l} \leqq P<j_{l+1}$, and let

$$
\begin{aligned}
R_{i, r}^{l} & =\left\{e \in R_{i, r}: j_{l+1}>\sum_{s=k}^{l} e_{s} \geqq j_{l}\right\}, \\
R_{i, r}^{l}(P) & =\left\{e \in R_{i, r}^{l}: \sum_{s=k}^{l} e_{s}=P\right\} .
\end{aligned}
$$

Clearly if $j_{r} \leqq i \leqq j_{r+1}$, then

$$
\bigcup_{l=n_{N}}^{r} R_{i, r}^{l}=R_{i, r} \quad \text { and } \bigcup_{P=j_{l}}^{j_{l+1}-1} R_{i, r}^{l}(P)=R_{i, r}^{l}
$$

and therefore

$$
\sum_{e \in R_{i, r}} \prod_{s=k}^{n} \lambda_{s}^{e_{s}}\left(\begin{array}{c}
i-\sum_{t=k}^{s-1} e_{t} \\
e_{s}
\end{array}\right) \leqq \sum_{l=n_{N}}^{r} \sum_{P=j_{l}}^{j_{l+1}-1} \sum_{e \in R_{i, r}^{l}(P)} \prod_{s=k}^{n} \lambda_{s}^{e_{s}}\left(\begin{array}{c}
i-\sum_{t=k}^{s-1} e_{t} \\
e_{s}
\end{array}\right) .
$$

But an easy computation shows that

$$
\sum_{e \in R_{i, r}^{l}(P)} \prod_{e=k}^{n} \lambda_{s}^{e_{s}}\left(\begin{array}{c}
i-\sum_{t=k}^{s-1} e_{t} \\
e_{s}
\end{array}\right)=\left(\begin{array}{l}
i \\
P
\end{array}\right)\left(\sum_{s=k}^{i} \lambda_{s}\right)^{P}\left(\sum_{s=l+1}^{n} \lambda_{s}\right)^{i-P}
$$


This implies that

$$
\begin{aligned}
\sum_{r=n_{N}}^{n} \sum_{i=j_{r}}^{j_{r}+1}-1 & (1 / i !) \sum_{e \in R_{l, r}} \prod_{s=k}^{n} \lambda_{s}^{e_{s}}\left(\begin{array}{c}
i-\sum_{t=k}^{s-1} e_{t} \\
e_{s}
\end{array}\right) \\
& \leqq \sum_{r=n_{N}}^{n} \sum_{i=j_{r}}^{j_{r}+1-1}(1 / i !) \sum_{l=n_{N}}^{r} \sum_{P=j_{l}}^{j_{l}+1-1}\left(\begin{array}{l}
i \\
P
\end{array}\right)\left(\sum_{s=k}^{l} \lambda_{s}\right)^{P}\left(\sum_{s=l+1}^{n} \lambda_{s}\right)^{i-P} \\
& =\sum_{l=n_{N}}^{n} \sum_{P=j_{l}}^{j_{l}+1-1}(1 / P !)\left(\sum_{s=k}^{l} \lambda_{s}\right)^{P} \sum_{i=P}^{j_{n}+1-1}\left(\sum_{s=l+1}^{n} \lambda_{s}\right)^{i-P} /(i-P) ! \\
& \leqq \sum_{l=n_{N}}^{n}\left(\sum_{s=k}^{l} \lambda_{s}\right)^{j_{l}} / j_{l} ! \exp \left(\sum_{s=k}^{l} \lambda_{s}\right) \exp \left(\sum_{s=l+1}^{n} \lambda_{s}\right)^{-1} \\
& <(\varepsilon / 2)
\end{aligned}
$$

which clearly completes the proof of the lemma.

The next three lemmas and the conditions on the $b_{n}$ 's are taken from Herman Rubin's technical report [5]. The statements of lemmas three and four and the methods used in their proofs appear here essentially as they appear in his report, and are included here for two reasons: 1 . for completeness, and 2 . because the source is relatively obscure. The statement of lemma five is presented here as it is found in his report, except that I found I needed the additional hypothesis that $\lim \sup _{n} b_{n} / b_{n+1}=0$. His proof appears to be incorrect, and I present here a proof which is probably what Rubin had in mind.

In the next three lemmas, let $\left\{b_{n}\right\} \subset I^{+}$be such that for $n$ sufficiently large: (1) $b_{n+3} / b_{n}>k>1$, and (2) $b_{n+1}-b_{n} \geqq n$. Also let $N$ be an infinite collection of positive integers.

Let $x \in(-\infty, \infty)$, let $x$ be expanded into its binary form, and let $\pi_{i}$ map $x$ onto the zero or the one appearing in the $i$ th place to the right of the decimal. Let $n \in I^{+}$, and let

$$
\begin{aligned}
& A_{n}=\left\{x \in(-\infty, \infty): \pi_{i} x=0 \text { for } b_{n} \leqq i \leqq b_{n-1}-[n / 2]\right\} \\
& A_{n}^{\prime}=\left\{x \in(-\infty, \infty): \pi_{i} x=0 \text { for } b_{n} \leqq i \leqq b_{n+1}-[n / 2]-1\right\}
\end{aligned}
$$

Now lemmas three and four are stated in terms of $A_{n}$, but it is quite clear that they are still true lemmas if $A_{n}$ is replaced by $A_{n}^{\prime}$.

LEMMA 3. $\left|\bigcap_{m=1}^{\infty}\left(\bigcup_{n=m ; n \in N}^{\infty} A_{n}\right)\right|=0$, and

$$
\operatorname{dim}\left(\bigcap_{m=1}^{\infty}\left(\bigcap_{n=m \in N}^{\infty} A_{n}\right)\right) \leqq \limsup _{n \in N} b_{n} / b_{n+1} .
$$

Proof. Let $A=\bigcap_{m=1}^{\infty}\left(\bigcup_{n=m: n \in N}^{\infty} A_{n}\right)$. By the countable additivity of measures and A.1 in $\S I$ all we need show in order to prove this lemma is that if $l$ is an integer, then $|A \cap[l, l+1]|=0$ and $\operatorname{dim}(A \cap[l, l+1]) \leqq \lim \sup _{n \in N} b_{n} / b_{n+1}$. 
Now $A \cap[l, l+1] \subset \bigcup_{n=m ; n \in N}^{\infty}\left(A_{n} \cap[l, l+1]\right)$ for every $m \in I^{+}$by the definition of $A$. But note that $A_{n} \cap[l, l+1]$ can be covered by $2^{b_{n}}$ intervals of length $2^{-\left(b_{n+1}-[n / 2]\right)}$. So we first see that for every $m \in I^{+}$

$$
|A \cap[l, l+1]| \leqq \sum_{n=m}^{\infty} 2^{b_{n}-b_{n+1}+[n / 2]} \leqq \sum_{n=m}^{\infty} 2^{-n / 2}
$$

by assumption (2) on the $b_{n}$ 's. But $\sum_{n=1}^{\infty} 2^{-n / 2}$ is a convergent series and therefore $|A \cap[l, l+1]|=0$. Secondly, we see, by the definition of Hausdorff dimension, that if $\alpha$ is a real positive number and

$$
\sum_{n \in N} 2^{b_{n}-\alpha\left(b_{n+1}-[n / 2]\right)}<\infty
$$

then $\operatorname{dim}(A \cap[l, l+1]) \leqq \alpha$. So we let $\alpha>\lim \sup _{n \in N} b_{n} / b_{n+1}$ and shall show this series converges. Let $\varepsilon>0$ be such that

$$
(1-\varepsilon) \alpha>\limsup _{n \in N} b_{n} / b_{n+1} .
$$

There exists $N_{1} \in I^{+}$such that $n \geqq N_{1}$ implies $b_{n}<b_{n+1}(1-\varepsilon) \alpha$. By condition (2) on the $b_{n}$ 's

$$
b_{n+1} \geqq \sum_{i=1}^{n} i=(n+1) n / 2 ;
$$

so there exists $N_{2} \in I^{+}$such that $n \geqq N_{2}$ implies

$$
b_{n+1}-[n / 2]>(1-\varepsilon / 2) b_{n+1} .
$$

By an easy computation using assumption (1) on the $b_{n}$ 's, we see there exists $N_{3} \in I^{+}$such that $n \geqq N_{3}$ implies $b_{n} \geqq H k_{0}^{n}$ where $H=\max \left(b_{1}, b_{2}, b_{3}\right)$ and $k_{0}=k^{1 / 4}$.

So if $n \geqq \max \left(N_{1}, N_{2}, N_{3}\right)$ then

$$
\begin{aligned}
b_{n}-\alpha\left(b_{n+1}-[n / 2]\right) & \leqq(1-\varepsilon) \alpha b_{n+1}-(1-\varepsilon / 2) \alpha b_{n+1} \\
& =-\varepsilon \alpha b_{n+1} / 2<-\varepsilon \alpha H k_{0}^{n+1} / 2 .
\end{aligned}
$$

By condition (1) on the $b_{n}$ 's, $k_{0}>1$, and therefore

$$
\sum_{n \in N} 2^{b_{n}-\alpha\left(b_{n+1}-[n / 2]\right)}<\infty
$$

and the proof of lemma three is complete.

Definition. We say a random variable $X$ is of Rubin type with Poissons $\left\{\lambda_{n}\right\}$ and partition $\left\{c_{n}\right\}$ if

$$
X=\sum_{n=1}^{\infty} \sum_{i=1}^{N_{n}} \sum_{j=c_{n}+1}^{c_{n}+1} A_{i j} / 2^{j},
$$

where $\left\{N_{n}, A_{i j}, i, j, n=1,2, \ldots\right\}$ are independent random variables, where the distribution of $N_{n}$ is Poisson with expectation $\lambda_{n}$, where each $A_{i j}$ takes values 0 and 1 with probability $1 / 2$ each, and where $\left\{c_{n}\right\}$ is an increasing sequence of positive 
integers. For notational convenience we let $N_{n}$ and $A_{i, j}$ be denoted by $N_{n}(X)$ and $A_{i, j}(X)$ respectively.

0.1 . Let $X_{1}, \ldots, X_{n}$ be a finite collection of independent random variables, with $X_{i}$ being of Rubin type with Poissons $\left\{\lambda_{i, k}\right\}$ and partition $\left\{c_{n}\right\}$ for $i=1, \ldots, n$. Let $Y=\sum_{i=1}^{n} X_{i}$ and let $F_{Y}$ be the distribution function of $Y$. Then by glancing at the characteristic function of $F_{Y}$ we see that $F_{Y}$ is the distribution function of a random variable of Rubin type with Poissons $\left\{\sum_{i=1}^{n} \lambda_{i, k}\right\}$ and partition $\left\{c_{n}\right\}$.

We note in passing that, by a result due to Hartman and Wintner [4], $X$ has a pure distribution.

In lemmas four and five we assume $X$ is of Rubin type with partition $\left\{b_{n}\right\}$ and Poissons $\left\{\lambda_{n}\right\}$ where

1. $\sum_{n=1}^{\infty} \lambda_{n}=\infty$, a condition which by [4] implies $X$ has a continuous distribution, and

2. $\lambda_{n} \leqq n$.

Lemma 4. If $\sum_{n \in N} \exp \left(-\lambda_{n}\right)=\infty$, then $\operatorname{Pr} .\left[X \in \bigcap_{m=1}^{\infty}\left(\bigcup_{n=m: n \in N} A_{n}\right)\right]=1$, and therefore

$$
\operatorname{dim} \operatorname{supp}(X) \leqq \limsup _{n \in N} b_{n} / b_{n+1}
$$

Proof. Claim 1. If $c$ is a positive real number, then $\operatorname{Pr} .\left[N_{n+1}(X) \geqq 2^{[n / 2]-c}\right.$ infinitely often $]=0$.

By the Borel-Cantelli lemma we see that in order to prove this claim we only need show that

$$
\sum_{n=1}^{\infty} \operatorname{Pr} .\left(N_{n+1}(X) \geqq 2^{[n / 2]-c}\right)<\infty
$$

But for each $n \in I^{+}, N_{n+1}(X)$ is a Poisson distributed random variable and therefore

$$
\operatorname{Pr} .\left(N_{n+1}(X) \geqq 2^{[n / 2]-c}\right)=\exp \left(-\lambda_{n+1}\right) \sum_{n=2^{[n / 2]}-c}^{\infty}\left(\lambda_{n+1}\right)^{j} / j !
$$

So by Taylor's Theorem with remainder we see that

$$
\operatorname{Pr} .\left(N_{n-1}(X) \geqq 2^{[n / 2]-c}\right) \leqq\left(\lambda_{n+1}\right)^{2^{[n / 2]-c}} /\left(2^{[n / 2]-c}\right) !
$$

Now combining assumption 2 on the $\lambda_{n}$ 's with the ratio test we see that our claim follows.

Using the Borel-Cantelli lemma again with the condition that $\sum_{n \in N} \exp \left(-\lambda_{n}\right)$ $=\infty$, we see that $\operatorname{Pr} .\left[N_{n}(X)=0\right.$ infinitely often $]=1$. Therefore there exists a set $M$ such that $\operatorname{Pr} .(M)=1$, and, if $\omega \in M$, then (1) there exists $N^{\prime}(\omega) \in I^{+}$such that if $n \geqq N^{\prime}(\omega)$ then $N_{n+1}(X)(\omega)>2^{[n / 2]-c} ;(2)$ there exists a sequence $\left\{n_{m}(\omega)\right\} \subset I^{+}$with $N_{n_{j}(\omega)}(X)(\omega)=0$ for every $j \in I^{+}$. In order to prove this lemma it is clearly sufficient to show that if $m \in I^{+}$then $\operatorname{Pr}$. $\left[X \in \bigcup_{n=m}^{\infty} A_{n}\right]=1$. So let $m \in I^{+}, \omega \in M$, and let 
$j \in I^{+}$be such that $n_{j}(\omega) \geqq \max \left(N^{\prime}(\omega), m\right)$; then we will show $X(\omega) \in A_{n_{j}(\omega)}$ which clearly implies $\operatorname{Pr} .\left[X \in \bigcup_{n=m}^{\infty} A_{n}\right]=1$. Now by assumption $N_{n,(\omega)}(X)(\omega)=0$ and therefore

$$
X_{n_{j}(\omega)}(\omega)=\sum_{n=1}^{n_{j}(\omega)-1} \sum_{n=n_{j}+1}^{\infty}\left(\sum_{i=0}^{N_{n}(X)(\omega)} \sum_{j=b_{n}+1}^{b_{n+1}} A_{i, j}(\omega) 2^{-j}\right) .
$$

But recall that $n_{j}(\omega) \geqq N^{\prime}(\omega)$ and therefore

$$
\begin{aligned}
\sum_{n=n_{j}(\omega)+1}^{\infty}\left(\sum_{i=0}^{N_{n}(X)(\omega)} \sum_{j=b_{n}+1}^{b_{n}+1} A_{i, j}(\omega) 2^{-j}\right) & \leqq \sum_{n=n_{j}(\omega)+1}^{\infty} 2^{[n / 2]-c}\left(\sum_{j=b_{n}+1}^{b_{n+1}+1} 2^{-j}\right) \\
& \leqq \sum_{n=n_{j}(\omega)+1}^{\infty} 2^{[n / 2]-b_{n}-c}<2^{\left[\left(n_{j}(\omega)+1\right) / 2\right]-b_{n_{j}(\omega)+1}} .
\end{aligned}
$$

Therefore $\pi_{i} X(\omega)=0$ for $b_{n_{j}(\omega)}<i \leqq b_{n_{j}(\omega)+1}-\left[\left(n_{j}(\omega)+1\right) / 2\right]$ which by definition implies $X(\omega) \in A_{n_{f}(\omega)}$.

LemMA 5. Let $\sum_{n \notin N} \exp \left(-\lambda_{n}\right)<\infty, \lim \sup _{m, n \in N: m<n} b_{m} / b_{n}=0$, and

then

$$
\limsup _{n \in I^{+}} b_{n} / b_{n+2}=0 \text {; }
$$

$$
\operatorname{dim} \operatorname{supp}(X) \geqq \liminf _{n \in N} b_{n} / b_{n+1} \text {. }
$$

Proof. Let

$$
\begin{aligned}
X_{i, n} & =\sum_{i=b_{n}+1}^{b_{n}+1} A_{i, j}(X) 2^{-j}, \\
V_{1} & =\sum_{n \notin N} X_{1, n}=\sum_{n \notin N ; N_{n}(X) \neq 0} X_{1, n}+\sum_{n \notin N ; N_{n}(X)=0} X_{1, n}, \\
Z & =\sum_{N_{n}(X) \neq 0} X_{1, n}=\sum_{n \in N: N_{n}(X) \neq 0} X_{1, n}+\sum_{n \notin N ; N_{n}(X) \neq 0} X_{1, n}, \\
W & =\sum_{n=1}^{\infty} \sum_{i=2}^{N_{n}(X)} X_{i, n}, \\
U & =W+\sum_{n \in N ; N_{n}(X) \neq 0} X_{1, n}+V_{1} .
\end{aligned}
$$

Now first we note $X=W+Z$. Secondly we note that $W+\sum_{n \in N: N_{n}(X) \neq 0} X_{1, n}$ and $V_{1}$ are independent random variables and therefore by A.3 dim supp $V_{1} \leqq \operatorname{dim} \operatorname{supp}(U)$. Note that

$$
\begin{aligned}
X+\sum_{n \notin N: N_{n}(X)=0} X_{1, n} & =W+Z+\sum_{n \notin N: N_{n}(X)=0} X_{1, n} \\
& =W+V_{1}+\sum_{n \in N: N_{n}(X) \neq 0} X_{1, n}=U
\end{aligned}
$$

But by hypothesis $\sum_{n \notin N} \exp \left(-\lambda_{n}\right)<\infty$, so by the Borel-Cantelli lemma

$$
\operatorname{Pr} .\left(N_{n}(X)=0 \text { for all } n \notin N \text {, except for finitely many }\right)=1
$$


which implies $\sum_{n \notin N: N_{n}(X) \neq 0} X_{1, n}$ is discrete since $X_{1, n}$ is discrete for every $n$. So by A.4

$$
\operatorname{dim} \operatorname{supp}(X)=\operatorname{dim} \operatorname{supp}(U) \text {. }
$$

So in order to prove this lemma, we see that we only need show that

$$
\operatorname{dim}\left(V_{1}\right) \geqq \liminf _{n \in N} b_{n} / b_{n+1} .
$$

A theorem proved by S. D. Chatterji [2] says that if $k \in I^{+}$, and if $\left\{X_{j}: j \in I^{+}\right\}$ is a collection of independent random variables such that each $X_{j}$ assumes values in $\{0,1,2, \ldots, k-1\}$, if $P_{i j}=\operatorname{Pr} .\left[X_{j}=i\right]$, and if $Y=\sum_{j=1}^{\infty} X_{j} / k^{j}$, then

$$
\operatorname{dim} \operatorname{supp}(Y)=\liminf _{n \rightarrow \infty}\left\{(-1 /(n \ln k)) \sum_{j=1}^{n}\left(\sum_{i=1}^{k} P_{i, j} \ln P_{i, j}\right)\right\}
$$

with the convention that $0 \ln 0=0$. Let $j(n)=\max \left\{i: b_{i} \leqq n\right\}$, and for $m \in I^{+}$let

$$
\begin{array}{rlrl}
D_{m} & =(1 / m)\left(\sum_{j \notin N ; j<j(m)}\left(b_{j+1}-b_{j}\right)+m-b_{j(m)}\right) & & \text { if } j(m) \notin N, \\
& =(1 / m)\left(\sum_{j \notin N: j<j(m)}\left(b_{j+1}-b_{j}\right)\right) & \text { if } j(m) \in N .
\end{array}
$$

By Chatterji's theorem we find that, by an easy computation,

$$
\operatorname{dim} \operatorname{supp}\left(V_{1}\right)=\liminf _{n \rightarrow \infty} D_{n} \text {. }
$$

Case 1. There exists $\left\{n_{i}\right\} \subset I^{+}$such that $n_{i} \in N$ and $n_{\imath}+1 \in N$.

By hypothesis $\lim \sup _{m, n \in N: m<n} b_{m} / b_{n}=0$ and therefore $\lim \sup _{i} b_{n_{i}} / b_{n_{i}+1}=0$ which implies lim inf $\operatorname{in}_{n \in N} b_{n} / b_{n+1}=0$ and the conclusion of the lemma holds.

Case 2. There exists $N_{0} \in I^{+}$such that $n \geqq N_{0}$ and $n \in N$ implies $n+1 \notin N$.

Let $\varepsilon>0$; then there exists $n_{\varepsilon} \in I^{+}$such that $n \geqq n_{\varepsilon}$ and $n \in N$ imply

$$
b_{n} / b_{n+1}>\liminf _{n \in N} b_{n} / b_{n+1}-\epsilon / 2 \text {. }
$$

By hypothesis $\lim \sup _{n} b_{n} / b_{n+2}=0$ and therefore there exists $n^{\prime}(\varepsilon)$ such that $n \geqq n^{\prime}(\varepsilon)$ implies $b_{n} / b_{n+2}<\varepsilon / 2$. Let $N(\varepsilon)=\max \left(N_{0}, n_{\varepsilon}, n^{\prime}(\varepsilon)\right)$. Let

$$
j^{\prime}(n)=\max \left\{i: b_{i} \leqq n, i \in N\right\} .
$$

Now $N$ is an infinite set by assumption and therefore $j^{\prime}(n) \underset{n}{\rightarrow} \infty$. So there exists $N^{\prime}(\varepsilon)$ such that $n \geqq N^{\prime}(\varepsilon)$ implies $j^{\prime}(n) \geqq N(\varepsilon)$. Let $n \geqq \max \left(N(\varepsilon), N^{\prime}(\varepsilon)\right)$.

Case $i$. $j(n) \notin N$. In this case

$$
\begin{aligned}
D_{n} & =(1 / n)\left(\sum_{j \notin N: j<j(n)}\left(b_{j+1}-b_{j}\right)+\left(n-b_{j(n)}\right)\right) \\
& =(1 / n)\left(n-b_{j^{\prime}(n)+1}+\sum_{j \notin N: j<j^{\prime}(n)}\left(b_{j+1}-b_{j}\right)\right) .
\end{aligned}
$$


Note that if $R, S \in I^{+}$, then $R / S<(R+1) /(S+1)$, so applying this inequality $n-b_{j^{\prime}(n)+1}$ times to $D_{n}$ we see

$$
D_{n} \geqq\left(1 / b_{j^{\prime}(n)+1}\right) \sum_{j \notin N ;}\left(b_{j<j^{\prime}(n)}-b_{j}\right) .
$$

Therefore recalling that $j^{\prime}(n) \geqq N(\varepsilon)$ and $j^{\prime}(n) \varepsilon N$, we obtain

$$
\begin{aligned}
D_{n} & \geqq\left(b_{j^{\prime}(n)}-b_{j^{\prime}(n)-1}\right) / b_{j^{\prime}(n)+1} \\
& \geqq b_{j^{\prime}(n)} / b_{j^{\prime}(n)+1}-\varepsilon / 2 \geqq \lim _{n \in N} \inf _{n} / b_{n+1}-\varepsilon .
\end{aligned}
$$

Case ii. $j(n) \in N$. By hypothesis of Case 2 , we see that $j(n)-1 \notin N$ and therefore

$$
D_{n}=(1 / n) \sum_{j \notin N: j<j(n)}\left(b_{j+1}-b_{j}\right) \geqq\left(b_{j(n)}-b_{j(n)-1}\right) / b_{j(n)+1}
$$

Now recall that $j(n) \geqq N(\varepsilon)$ and $j(n) \in N$, so

$$
D_{n} \geqq b_{j(n)} / b_{j(n)+1}-\varepsilon / 2 \geqq \liminf _{n \in N} b_{n} / b_{n+1}-\varepsilon .
$$

In both Case $\mathrm{i}$ and Case ii, $\varepsilon$ was arbitrary, and so the proof of the lemma is complete.

LeMma 6. Let $m:[0, \infty) \rightarrow[0,1]$ such that

1. $m(0)=1$,

2. $m(t) m(d) \geqq m(t+d)$ for $t, d \in[0, \infty)$, and

3. there exists $\alpha>0$ such that $m(t) \neq 0$ for $t \in[0, \alpha]$. Then the right-hand derivative of $m$ exists at 0 , and we call it $m^{\prime}(0)$, and $\ln m(t) / t \rightarrow m^{\prime}(0)$ as $t \rightarrow 0^{+}$.

Proof. Now clearly, by induction, $m(t) m(d) \geqq m(t+d)$ implies that $m^{n}(x / n)$ $\geqq m(x)$, and therefore

$$
-\ln m(x / n) \leqq-(\ln m(x)) / n .
$$

Let $g=-\ln m(x)$ for $x \in[0, \alpha]$. By hypothesis, $m(t) \leqq 1$ for every $t \geqq 0$, so this combined with $m(t) m(d) \geqq m(t+d)$ implies that $m$ is a nonincreasing function. Since $m(t) \neq 0$ on $[0, \alpha]$ there exists $c>0$ such that $1 \geqq c$ and $m(t) \rightarrow c$ as $t \rightarrow 0+$. We will now show that $c=1$, i.e. that $m$ is continuous from the right at 0 . Suppose $c<1$. Then choose $t \in[0, \alpha]$ and let $n$ be such that $m(t)>c^{n}$. It was already noted that $m^{n}(t / n) \geqq m(t)$. But $m$ is nondecreasing and therefore $c \geqq m(t / n)$ which implies $c^{n} \geqq m(t)$; this is a contradiction.

In Bruckner and Ostrow [1, Section 4], one finds the following theorem:

THEOREM. Let $f$ be a continuous nonnegative function on $[0, c]$ such that $f(0)=0$ and $f(x / n) \leqq(1 / n) f(x)$ for $x \in[0, c]$. Then $f$ is differentiable at 0 .

Now if one examines the proof that Bruckner and Ostrow give for this theorem, one sees that they really only use the continuity of $f$ at 0 and on some countable sequence of points which converges to 0 . We have already shown that our function 
$m$ is continuous at 0 , and since $m$ is monotone nonincreasing it is continuous at all but a countable set of points and therefore certainly continuous on a sequence of points converging to 0 . But note that the function $g$ is clearly continuous if $m$ is, $g$ is nonnegative since $m(x) \leqq 1, g(0)=-\ln 1=0$, and as was seen at the beginning of the proof, $g$ satisfies the inequality in the theorem of Bruckner and Ostrow. So $g$ has a derivative at 0 , which in turn implies that $m$ has a derivative at 0 . Now

$$
\ln m(x) / x=\left(\int_{1}^{m(x)} d t / t\right) / x .
$$

Therefore, by the mean value theorem for integrals, there exists $t_{0}$, where $1 \leqq t_{0}$ $\leqq m(x)$, such that

So

$$
\ln m(x) / x=(m(x)-1) /\left(t_{0} x\right) .
$$

$$
(m(x)-1) / x \leqq \ln m(x) / x \leqq(m(x)-1) /(x m(x)) .
$$

But $m$ is continuous at 0 and $m(0)=1$, so taking limits across the above inequalities, we see that

$$
\lim _{x \rightarrow 0+} \ln m(x) / x=m^{\prime}(0)
$$

which completes the proof of this lemma.

Lemma 7. Let a function $f(t)$ map $(-\infty, \infty)$ into $(-\infty, \infty)$. Then there exists a countable set $D \subset(-\infty, \infty)$ such that if $x \in(-\infty, \infty)$, then there exists a sequence $\left\{x_{j}\right\} \subset D$ where $x_{j} \rightarrow x+$ and $f\left(x_{j}\right) \rightarrow f(x)$ as $j \rightarrow \infty$.

Proof. Let $r$ and $s$ be two rational numbers, and let

$$
D_{r, s}=\{t: r<f(t)<s\} .
$$

Let $T_{r, s}$ be the set of all points of $D_{r, s}$ which are isolated from the right relative to $D_{r, s}$. Clearly this is a countable set. Now $D_{r, s}$ is a subset of the real numbers, and therefore there exists a countable dense subset of $D_{r, s}$, call it $D_{r, s}^{\prime}$ Let $E_{r, s}=T_{r, s}$ $\cup D_{r, s}^{\prime}$ and let

$$
D=\bigcup_{r, s \in \mathrm{Ra}} E_{r, s}
$$

where $\mathrm{Ra}$ is the set of rational numbers.

Now we will show that this set $D$ is the set desired. Let $x \in(-\infty, \infty)$. If $x \in D$, then let $x_{j}=x$ for every $j \in I^{+}$and the conclusion to this lemma is proved. If $x \in(-\infty, \infty)-D$, then let $\left(s_{i}, r_{i}\right)$ be a sequence of intervals such that $s_{i}, r_{i} \in \mathrm{Ra}$ for every $i \in I^{+},\left(s_{i+1}, r_{i+1}\right) \subset\left(s_{i}, r_{i}\right),\{f(x)\}=\bigcap_{i}\left(s_{i}, r_{i}\right)$ and $r_{i}-s_{i}<1 / i$. Now clearly by the definition of $D_{r_{i}, s_{i}}, x \in D_{r_{i}, s_{i}}$ for every $i \in I^{+}$. Now $x \in(-\infty, \infty)-D$, so $x$ certainly is not isolated from the right relative to $D_{r_{i}, s_{i}}$ by the definition of $D$. So for each $i$, let $\left\{x_{i, j}\right\} \subset D_{r_{i}, s_{i}}$ such that $x_{i, j} \rightarrow x+$ for each $i \in I^{+}$. But there exists, for each $i$, an integer $j(i)$ such that $x_{i, j(i)} \in(x, x+1 / i)$, and let $x_{i}=x_{i, j(i)}$. By the definition of $D_{r_{i}, s_{i}}$

$$
\left|f(x)-f\left(x_{i}\right)\right|<1 / i
$$

and therefore $f\left(x_{i}\right)_{i} \rightarrow f(x)$ which completes the proof of this lemma. 
LEMmA 8. Let $\left\{G_{t}(x): t \in(0, \infty)\right\}$ be a collection of distribution functions such that for fixed $x, G_{t}(x)$ decreases for increasing $t$. Let $F$ be a distribution function and $\lambda$ a bounded nonincreasing function on $(0, \infty)$; then for $i \in I^{+}$

$$
\left(\int_{0}^{\infty} G_{\tau} d \lambda(\tau)\right)^{* i} * F(x)=\int_{0}^{\infty} \cdots \int_{0}^{\infty} \underset{j=1}{*} G_{\tau_{j}}^{i} * F(x) d \lambda\left(\tau_{1}\right) \cdots d \lambda\left(\tau_{n}\right) .
$$

Let $G(t, x) \doteq G_{t}(x)$. Note that $G(t, x)$ is a distribution function in $x$ for fixed $t$, and for fixed $x$ it decreases with increasing $t$ by assumption. Standard argunents imply then that $G(t, x)$ is jointly measurable. Induction and Fubini's theorem quickly yield the lemma.

We now prove the theorem stated at the beginning of this section.

Proof. We shall first prove this theorem for functions $m(t)$ such that $A$ and $B$ are both empty, and then we shall indicate how to modify the definitions and arguments for the general case. The reason for this approach is that all the main ideas and arguments are used in this case, and yet we are not burdened down with superfluous cases and lengthy expressions which only tend to obscure what is really going on.

So let $m(t)$ and $v(t)$ be functions as assumed in the hypothesis with the added condition that $A=B=\varnothing$. Let $D_{1}$ be a countable set contained in $(0, \infty)$ such that if $t \in(0, \infty)$ then there exists $\left\{t_{i}\right\} \subset D_{1}$ such that $t_{i} \rightarrow t^{+}$and $v\left(t_{i}\right) \rightarrow v(t)$. The existence of $D_{1}$ is of course guaranteed to us by Lemma 7 . Let

$$
D_{2}=\left\{t \in(0, \infty): m(t) \neq m\left(t^{+}\right)\right\} .
$$

By the monotonicity of $m$, we see that $D_{2}$ is countable. Let

$$
D_{3}=\left\{t \in(0, \infty): \liminf _{s \rightarrow t} v(s) \neq v(t)\right\} .
$$

By Lemma 1.2 and by hypothesis, we see that $D_{3}$ is countable. Let

$$
D=D_{1} \cup D_{2} \cup D_{3} \text {. }
$$

Let $\left\{c_{i}\right\}$ be a sequence of positive integers such that $c_{i} \downarrow 1$. Now clearly $D$ is countable, and $D$ is enumerated as follows:

Let $d_{1}$ be any element of $D$. Suppose $d_{n}$ has been defined, and let $d_{n+1} \in D$ be such that for $j=1, \ldots, n$ either $d_{n+1} / d_{j}>c_{n+1}$ or $d_{j} / d_{n+1}>c_{n+1}$. Denote

$$
l_{1}=\min \left\{j: \sum_{i=1}^{j} 1 / 4 i \geqq 1\right\} \text {. }
$$

Suppose $l_{n}$ has been defined; then define

Define

$$
l_{n+1}=\min \left\{j: \sum_{i=l_{n}+1}^{j} 1 / 4 i \geqq 1\right\} \text {. }
$$

$$
I_{n}=\left\{4 i: l_{n-1} \leqq i<l_{n}\right\} \text {. }
$$


Let $P_{1}, P_{2}, \ldots$ be an enumeration of the primes. Let $i \in I^{+}$, and let $\{l(i, j)\}_{j=1}^{\infty}$ be a sequence of increasing integers such that

Define

$$
\sum_{j=1}^{\infty} \sum_{n \in P_{i} l(i, j)} 1 / n^{c_{j+i}}<1 / 2^{i}
$$

$$
\bar{P}_{i}=\bigcup_{j} I_{P_{i} l(i, j)}, \quad P=\bigcup_{i} \bar{P}_{i}
$$

Clearly $\bar{P}_{i} \cap \bar{P}_{j}=\varnothing$ if $i \neq j$, and if $n, m \in P$, then $|n-m| \geqq 4$. If $t \in[0, \infty)$ we denote

$$
\begin{array}{rlrl}
\lambda(t) & =-\ln m(t) / t & & \text { if } t \neq 0 \\
& =-m^{\prime}(0) & \text { if } t=0 .
\end{array}
$$

By Lemma 6 and the hypothesis we see that $\lambda$ is a bounded nondecreasing function on all finite intervals of $[0, \infty)$ and is continuous at 0 .

Let $\left\{v_{n}\right\}$ be a sequence of real numbers such that $v_{n} \uparrow+\infty$, and for convenience let $v_{0}=0$. If $t \in(0, \infty)$, then let us denote

$$
[t]^{*}=\max \left\{n \in I^{+} \cup\{0\}: v_{n} \leqq t\right\} .
$$

If $n \in I^{+}$and $t \in(0, \infty)$ we define

$$
\begin{array}{rlrl}
t(n) & =v_{n-1} & & \text { if } n \leqq[t]^{*}+1, \\
& =t & & \text { if } n=[t]^{*}+2, \\
& =v_{n-2} & & \text { if } n \geqq[t]^{*}+3, \\
t^{\prime}(n) & =v_{n} & & \text { if } n \leqq[t]^{*}, \\
& =t & & \text { if } n=[t]^{*}+1, \\
& =v_{n-1} & \text { if } n \geqq[t]^{*}+2, \\
\lambda_{n}^{t}(x) & =\lambda(t(n)) & & \text { if } x \leqq t(n), \\
& =\lambda(x) & & \text { if } t(n) \leqq x \leqq t^{\prime}(n), \\
& =\lambda\left(t^{\prime}(n)\right) & & \text { if } x \geqq t^{\prime}(n) .
\end{array}
$$

Let $\lambda_{n}^{t}=\mathrm{T}$.V. $\left(\lambda_{n}^{t}(x)\right)$, and therefore clearly $\lambda_{n}^{t}=\lambda\left(t^{\prime}(n)\right)-\lambda(t(n))$. Let $\{k(n)\}$ be a sequence of positive integers such that $k(n) \uparrow \infty$, and such that

$$
\sum_{n} \lambda\left(v_{k(n)}\right) / 2^{n}<\infty
$$

Let $\left\{j_{n}\right\}$ be a sequence of positive integers such that $\min \left(j_{n}, j_{k(n)}\right)>n$ and

$$
\sum_{n=1}^{\infty}\left(\lambda\left(v_{n}\right)\right)^{j_{n}} / j_{n} !<\infty .
$$


Define $j(l)=j_{r}$ where $l \in \bar{P}_{j}$ and $d_{j} \in\left[v_{r-1}, v_{r}\right]$. Let $j^{\prime}(l)=\max \left(j(l), j_{l}\right)$. Let $\left\{a_{n}\right\}$ be a sequence of positive integers such that if $k \in I^{+}$, then

$$
\sum_{l \in P: l \geqq k}\left(2 j^{\prime}(l) \ln l\right)^{2^{a} l} / 2^{a_{l}} !<\min \left\{1 / 2^{k} \lambda\left(v_{k+1}\right), 1 / 2^{k}\right\} .
$$

Finally we define a sequence of positive integers $\left\{b_{n}\right\}$ as follows:

Case 1. $n \notin P$.

Subcase (i). If $n-1 \notin P$, then $b_{n}=\left(b_{n-1}+1\right) n$.

Subcase (ii). If $n-1 \in \bar{P}_{j}$

$$
\begin{aligned}
& b_{n}=\left[b_{n-1} / v\left(d_{j}\right)\right]+n \quad \text { when } v\left(d_{j}\right) \neq 0 \text {, } \\
& =\left[b_{n-1} /(1 / n)\right]+n \quad \text { when } v\left(d_{j}\right)=0 \text {. }
\end{aligned}
$$

Case 2. If $n \in P_{j}$, let $b_{n}=\left(b_{n-1}+a_{n}+1\right) n$.

By a straightforward computation we see that these $b_{n}$ 's satisfy the conditions imposed on the $b_{n}$ 's in Lemmas 3, 4 and 5.

Claim 1. Let $X$ be a random variable of Rubin type with Poissons $\left\{\lambda_{n}\right\}$ and partition $\left\{b_{n}\right\}$. If $k \in I^{+}$and $\omega \in \bigcap_{n \geqq k+1 ; n \in P}\left[N_{n}(X)<2^{a_{n}}\right]$, then

$$
X_{k}(\omega)=\sum_{n \geqq k+1 ; n \in P} \sum_{l=0}^{N_{n}(X)(\omega)} \sum_{j=b_{n}+1}^{b_{n}+1} A_{l, j}(X)(\omega) 2^{-j}<2^{-b_{k}} .
$$

Proof. If $\omega \in \bigcap_{n \geqq k+1 ; n \in P}\left(N_{n}(X) \geqq 2^{a_{n}}\right)$ then by the definition of the $A_{i, j}(X)$ 's we see that:

$$
X_{k}(\omega)=\sum_{n \geqq k+1: n \in P} 2^{a_{n}}\left(\sum_{j=b_{n}+1}^{b_{n}+1} 2^{-j}\right) \leqq \sum_{n \geqq k+1: n \in P} 2^{a_{n}-b_{n}}
$$

By the definition of the $b_{n}$ 's we see that $a_{n}-b_{n}<-b_{n-1}-1$ which clearly completes the proof of Claim 1.

Let $G_{\tau}$ and $V_{t}$ be the distribution functions corresponding to the random variables $X_{\tau}$ and $Y_{t}$ which are of Rubin type with Poissons $\left\{\lambda_{n, \tau}\right\},\left\{\lambda_{n, t}^{\prime}\right\}$ respectively and common partition $\left\{b_{n}\right\}$, where

$$
\begin{array}{rlrl}
\lambda_{n, \tau} & =2 \ln n & & \text { if } n \in \bar{P}_{i}, d_{i}>\tau, n>j_{\left[d_{i}\right]^{*}} \\
& =0 & \text { otherwise, } \\
\lambda_{n, t}^{\prime} & =\left(t / d_{j}\right) \ln n & \text { if } n \in \bar{P}_{j}, n>j_{\left[d_{j}\right]^{*}} \\
& =2 \ln n & \text { otherwise. }
\end{array}
$$

We assume that $\left\{N_{n}\left(X_{t}\right), N_{n}\left(Y_{t}\right), A_{i, j}\left(X_{t}\right), A_{i, j}\left(Y_{t}\right): i, j, n \in I^{+}, t \in(0, \infty)\right\}$ is a collection of independent random variables.

Claim 2. For fixed $x, G_{t}(x)$ decreases as $t$ increases.

Proof. If $t_{2}>t_{1}$, then clearly by considering the characteristic function of $G_{t_{1}}$ 
and $G_{t_{2}}$ we see that $G_{t_{2}}=G_{t_{1}} * F$ where $F$ is the distribution function of a random variable of Rubin type with Poissons $\left\{\lambda_{n}\right\}$ and partition $\left\{b_{n}\right\}$, where

$$
\begin{aligned}
\lambda_{n} & =2 \ln n & & \text { if } n \in \bar{P}_{i}, n \geqq j_{\left[d_{i}\right]}, t_{2}>d_{i} \geqq t_{1}, \\
& =0 & & \text { otherwise. }
\end{aligned}
$$

Therefore $G_{t_{2}}(x)=\int_{0}^{x} G_{t_{1}}(x-y) d F(y) \leqq G_{t_{1}}(x)$ which establishes Claim 2 .

Claim 2 shows that for fixed $x, G_{t}(x)$ is a Borel measurable function of $t$. So, for $x>0$ and $t \in[0, \infty)$, let

$$
\begin{aligned}
M_{n}^{t}(x) & =\int_{t(n)}^{t^{\prime}(n)} G_{\tau}(x) d \lambda_{n}^{t}(\tau)-\lambda_{n}^{t} & & \text { if } n \in I^{+}, \\
& =-m^{\prime}(0) V(x)+m^{\prime}(0) & & \text { if } n=0,
\end{aligned}
$$

where $V(x)$ is any absolutely continuous distribution function. Note that $M_{n}^{t}(x)$ increases as $x$ increases and $M_{n}^{t}(x) \leqq 0$ for every $x \in(0, \infty)$.

Claim 3. If $m \in I^{+}$, if $t \geqq v_{m}$ and if $x \geqq 1 / 2^{m}$, then

$$
G_{t}(x)>1-1 /\left(2^{[t]^{*}} \lambda\left([t]^{*}+1\right)\right) .
$$

Proof. Recalling the definition of $\lambda_{n, t}$ and noting that the condition $j_{n+1}>j_{n}$ implies $j_{\left[t^{*}\right.} \leqq j_{\left[d_{j}\right]^{*}}$ for $d_{j} \geqq t$, we see that for all $\omega$,

$$
X_{t}(\omega)=\sum_{n \geqq j_{[t]^{*}+1 ; n \in P}} \sum_{i=1}^{N_{n}\left(X_{t}\right)(\omega)} \sum_{j=b_{n}+1}^{b_{n+1}} A_{i, j}(X t)(\omega) 2^{-j} .
$$

Therefore by Claim 1, if $\omega \in \bigcap_{n \geqq j_{[t]^{*}+1 ; n \in P}}\left(N_{n}\left(X_{t}\right) \leqq 2^{a_{n}}\right)$ then $X_{t}(\omega)<1 / 2^{b_{f(t]^{*}}}$.

Since $b_{n}$ and $j_{n}$ are both greater than or equal to $n$, and $x \geqq 1 / 2^{m}$, we see that

Therefore

$$
x \geqq 1 / 2^{b_{f[t]^{*}}} \text {. }
$$

$$
\begin{aligned}
1-G_{t}(x) & =\operatorname{Pr} .\left(X_{t} \in(x, \infty)\right) \leqq \operatorname{Pr} .\left(X_{t} \in\left(1 / 2^{b_{f t t^{*}}}, \infty\right)\right) \\
& \leqq \sum_{n \geqq f_{[t]^{*+1}} ; n \in P} \operatorname{Pr} .\left(N_{n}\left(X_{t}\right)>2^{a_{n}}\right) .
\end{aligned}
$$

But the $N_{n}\left(X_{t}\right)$ 's are Poisson random variables and therefore

$$
1-G_{t}(x) \leqq \sum_{n \geqq j_{[t]^{*+1}, n \in P}}(2 \ln n)^{2^{a_{n}}} / 2^{a_{n}} !
$$

By this last inequality and our assumptions on the $a_{n}$ 's we see the proof of Claim 3 is complete.

Claim 4. $\sum_{n=0}^{\infty} M_{n}^{0}(x)>-\infty$ for every $x \in(0, \infty)$.

Proof. Fix $x \in(0, \infty)$. Now there exists $l \in I^{+}$such that $x \geqq 1 / 2^{l}$.

So by Claim 3 we see that for $n \geqq l+1$

$$
\begin{aligned}
M_{n}^{0}(x) & =\int_{v_{n-1}}^{v_{n}} G_{\tau}(x) d \lambda_{n}^{0}(\tau)-\lambda_{n}^{0} \geqq \lambda_{n} /\left(2^{n-1} \lambda\left(v_{n}\right)\right) \\
& \geqq-1 / 2^{n-1} .
\end{aligned}
$$


But for $n \leqq l, M_{n}^{0}(x)$ is a bounded function on $(0, \infty)$ and therefore the proof of Claim 4 follows.

Let

$$
M(x)=\sum_{n=0}^{\infty} M_{n}^{0}(x)
$$

and by Claim $4, M(x)>-\infty$ for every $x \in(0, \infty)$.

Claim 5. $\int_{0+}^{1} x d M(x)<\infty$.

Proof. Now clearly $\int_{0+}^{1} x d M_{0}^{0}(x)<\infty$ since $M_{0}^{0}(x)$ is a bounded function on $(0, \infty)$. So in order to prove Claim 5 we only need show that $\int_{0+}^{1} x d M^{\prime}(x)<\infty$ where $M^{\prime}(x)=\sum_{n=1}^{\infty} M_{n}^{0}(x)$. Now

But note that

$$
\begin{aligned}
\int_{0+}^{1} x d M^{\prime}(x) & =\sum_{l=0}^{\infty} \int_{\left(1 / 2^{l+1}\right)+0}^{1 / 2^{l}} x d M^{\prime}(x) \\
& \leqq \sum_{l=0}^{\infty}\left(M^{\prime}\left(1 / 2^{l}+0\right)-M^{\prime}\left(1 / 2^{l+1}\right)\right) / 2^{l}
\end{aligned}
$$

$$
M^{\prime}(x)=\lim _{n}\left(\int_{0}^{v_{n}} G_{\tau}(x) d\left(\sum_{j=1}^{n} \lambda_{j}(\tau)\right)-\left(\lambda\left(v_{n}\right)-\lambda(0)\right)\right)
$$

which along with the fact that $M^{\prime}$ increases for increasing $x$ implies that if $\varepsilon>0$, then

$$
\begin{aligned}
\int_{0+}^{1} x d M(x) & \leqq \sum_{l=0}^{\infty}\left(\int_{0}^{\infty}\left(G_{\tau}\left(1 / 2^{l}+\varepsilon\right)-G_{\tau}\left(1 / 2^{l+1}\right)\right)\right) d\left(\sum_{n=1}^{\infty} \lambda_{n}^{0}(\tau) / 2^{l}\right) \\
& \leqq \sum_{l=0}^{\infty}\left(\lambda\left(v_{k(l)}\right)+\int_{v_{k(l)}}^{\infty}\left(1-G_{\tau}\left(1 / 2^{l+1}\right)\right)\right) d\left(\sum_{n=k(l)+1}^{\infty} \lambda_{n}^{0}(\tau) / 2^{l}\right) .
\end{aligned}
$$

Recall that in the definition of $j_{n}$ we required that $j_{k(l)} \geqq 1$, which along with Claim 3 implies

$$
\begin{aligned}
\int_{v_{k(l)}}^{\infty} & \left(1-G_{\tau}\left(1 / 2^{l+1}\right)\right) d\left(\sum_{n=k(l)+1}^{\infty} \lambda_{n}^{0}(\tau)\right) \\
& \leqq \int_{n=k_{l}+1}^{\infty} \int_{v_{n-1}}^{v_{n}}\left(1-\left(1-1 /\left(2^{n-1} \lambda\left(v_{n}\right)\right)\right)\right) d \lambda_{n}^{0}(\tau) \\
& \leqq 1 / 2^{k_{l}-2} .
\end{aligned}
$$

This estimate along with the assumption on the $k(l)$ 's that $\sum_{l} \lambda\left(v_{k(l)}\right) / 2^{l}<\infty$ completes the proof of Claim 5 .

By Claims 4 and 5 we see that $M$ is a possible $M$-function in the Lévy representation of infinitely divisible distribution functions. Let $t \in(0, \infty)$ be fixed. Now we let $F_{t}$ be the distribution function corresponding to the characteristic function:

Now we let

$$
\text { ch.fcn. } V_{t} \cdot \exp \left(t \int_{0+}^{\infty}(\exp (i u x)-1) d M(x)\right) \text {. }
$$

$$
F_{t, n}(x)=\exp \left(-\sum_{i=0}^{n} \lambda_{i} t\right)\left(\sum_{i=0}^{\infty}\left(\sum_{j=0}^{n} M_{j}^{0}\right)^{* i} * V_{t} t^{i} / i !\right)(x) .
$$


Recalling that $\sum_{i=0}^{n} M_{i}(x) \underset{n}{\rightarrow} M(x)$, then a glance at the characteristic function for $F_{t, n}$ shows us that by [3], $F_{t, n} \stackrel{(c)}{\rightarrow} F_{t}$. Note that since $M_{0}$ is absolutely continuous,

$$
\left(F_{t, n}\right) \text { c.s. }=\exp \left(-t \sum_{i=0}^{n} \lambda_{i}\right) \sum_{i=0}^{\infty}\left(\left(\sum_{j=1}^{n} M_{j}\right)^{* i} * V_{t}\right) \text { c.s. } t^{i} / i ! .
$$

Claim 6. Let $\tau_{1}, \ldots, \tau_{i}$ be $i$ elements of $(0, \infty)$ and let $\tau_{0}=\min \left\{\tau_{j}: j \in\{1, \ldots, i\}\right\}$.

1. If $\tau_{0}<t$, then $*_{j=1}^{i} G_{\tau_{j}} * V_{t}$ is absolutely continuous.

2. If $t \leqq \tau_{0}$, and $\tau_{0} \geqq d_{i} \geqq t$, then

$$
\underset{j=1}{*} G_{\tau} * V_{t}\left[\bigcap_{n=1}^{\infty} \underset{n \geqq m: n \in P_{i}}{\bigcup_{n}} A_{n}\right]=1 \text {. }
$$

Proof. As noted before, $*_{j=1}^{i} G_{\tau_{j}} * V_{t}$ is the distribution function of a random variable of Rubin type with Poissons $\left\{\lambda_{n}^{\prime}\right\}$ and partition $\left\{b_{n}\right\}$ where $\lambda_{n}^{\prime}=\sum_{j=1}^{i} \lambda_{n, \tau_{j}}$ $+\lambda_{n, t}^{\prime}$. Now reviewing the definition of the $\lambda_{n, t}$ 's and the $\lambda_{n, t}^{\prime}$ 's we see that

$$
\begin{array}{rlrl}
\lambda_{n}^{\prime} & =\left(t / d_{j}\right) \ln n & & \text { if } n \in \bar{P}_{j}, n \geqq j_{\left[d_{j}\right]^{*}}, d_{j} \leqq \tau_{0}, \\
& \geqq 2 \ln n & \text { otherwise. }
\end{array}
$$

So if $d_{j} \leqq \tau_{0}<t$, then $t / d_{j} \geqq t / \tau_{0}>1$ and therefore clearly $\sum_{n} \exp \left(-\lambda_{n}^{\prime}\right)<\infty$. But a theorem due to Rubin [5] and easily accessible via Tucker [7] says that if $F$ is the distribution function of a random variable of Rubin type with Poissons $\left\{\lambda_{n}\right\}$ such that $\sum_{n} \exp \left(-\lambda_{n}\right)<\infty$, then $F$ is absolutely continuous. Therefore we see that if $\tau_{0}<t$ then indeed $*_{j=1}^{i} G_{\tau_{j}} * V_{t}$ is absolutely continuous. Since $\lambda_{n, \tau}$ and $\lambda_{n, t}^{\prime}$ are at most $2 \ln n$ for each $n \in I^{+}$, we see that $\lambda_{n}^{\prime} \leqq(j+1) \ln n$ and therefore for large enough $n, \lambda_{n}^{\prime} \leqq n$. So if $t \leqq d_{j} \leqq \tau_{0}$, then $t / d_{j} \leqq 1$. But by the way in which the $\bar{P}_{i}$ 's were defined we see that

$$
\sum_{n \in P_{1}} \exp \left(-\lambda_{n}^{\prime}\right) \geqq \sum_{n \in P_{1},} 1 / n=\infty .
$$

Therefore by Lemma 4 we see 2 of this claim is proved.

Let $T=\{\tau \in(0, \infty): \tau>t$ or $\tau=t$ if $t \in D\}$. By assumption on $D$, there exists $D_{0}=\left\{d_{i}^{\prime}\right\} \subset D$ such that $d_{i}^{\prime} \rightarrow t^{+}$and $v\left(d_{i}^{\prime}\right) \rightarrow v(t)$, and let us assume that if $t \in D$, then $t \in D_{0}$ also. Let $\tau \in T$. Let

$$
A(t, \tau)=\left\{n \in I^{+}: n \in \bar{P}_{i}, d_{i} \in D_{0}, t \leqq d_{i} \leqq \tau\right\} .
$$

Now by Claim 6

if $A(t, \tau) \neq \varnothing$.

$$
\underset{j=1}{*} G_{\tau_{j}} * V_{i}\left(\bigcap_{m=1}^{\infty} \bigcup_{n \geqq m ; n \in A(t, \tau)} A_{n}\right)=1
$$

Claim 7. Let $i \in I^{+}$; then

$$
\left(\left(\sum_{j=1}^{n+2} M_{j}^{t}\right)^{* i} * V_{t}\right) \text { c.s. }(x)=\left(\sum_{j=[t]+2}^{n+2} M_{j}^{t}\right)^{* i} * V_{t}(x) .
$$

For all $\tau \in T$ such that $A_{(t, \tau)} \neq \varnothing$,

$$
\left(\left(\sum_{j=1}^{n+2} M_{j}^{t}\right)^{* i} * V_{t}\right) \text { c.s. }\left(\bigcap_{n=1}^{\infty} \bigcup_{n \geqq m ; n \in A(t, \tau)} A_{n}\right)=(\lambda(n)-\lambda(t))^{i} .
$$


Proof.

$$
\left(\sum_{j=1}^{n+2} M_{j}^{t}\right)^{* i} * V_{t}=\left(\sum_{j=[t]+2}^{n+2} M_{j}^{t}\right)^{* i} * V_{t}+\sum_{l=1}^{i}\left(\begin{array}{l}
i \\
l
\end{array}\right)\left(\sum_{j=1}^{[t]+1} M_{j}^{t}\right)^{* l}\left(\sum_{j=[t]+2}^{n+2} M_{j}^{t}\right)^{* i-l} * V_{t}
$$

Now Claim 6, Lemma 8, and A.2 imply that every term in the second sum is absolutely continuous.

Lemma 9 implies

$$
\left(\sum_{j=[t]+1}^{n+2} M_{j}^{t}\right)^{* i} * V_{t}=\int_{t}^{n} \cdots \int_{t}^{n} \underset{j=1}{*} G_{\tau j} * V_{t} d \Lambda_{n}^{t} \cdots d \Lambda_{n}^{t}
$$

where $\Lambda_{n}^{t}=\sum_{l=[t]+2}^{n+2} \lambda_{l}^{t}$. Now if $m$ is continuous from the right at $t$, then $\lambda$ is continuous from the right at $t$, and we see we might as well be integrating from $t+0$ to $n$. If $m$ is not continuous from the right at $t$, then by definition of $D$ we see that $t \in D$. So by the remark just preceding this claim and A.2 we see that

$$
\left(\sum_{j=[t]+2}^{n+2} M_{j}^{t}\right)^{* i} * V_{t}\left(\bigcap_{m=1}^{\infty} \bigcup_{n \geqq m ; n \in A(t, \tau)} A_{n}\right)=(\lambda(n)-\lambda(t))^{i}
$$

if $A(t, \tau) \neq \varnothing$.

Now if $\tau>t$, then $A(t, \tau) \neq \varnothing$ and the above equality holds. But $A(t, \tau) \neq \varnothing$ implies $A(t, \tau)$ is an infinite subset of positive integers since $\bar{P}_{i}$ is an infinite subset of positive integers. So by Lemma 3

$$
\left|\bigcap_{m=1}^{\infty} \bigcup_{n \geqq m ; n \in A(t, \tau)} A_{n}\right|=0
$$

which completes the proof of Claim 7.

Clearly $M(x)=\sum_{i=0}^{\infty} M_{i}^{t}(x)$ and the $M_{i}^{t}$ 's satisfy the conditions imposed on the $M_{i}$ 's of Lemmas 1 and 2.

For $n>[t]^{*}$, we see that by Claim 7, the definition of the $M_{i}^{t}$ 's and the definition of $F_{t, n}$ that

$$
\left(F_{t, n}\right) \text { c.s. }(x)=\exp \left(-t \sum_{i=0}^{n+2} \lambda_{i}^{t}\right)\left\{\sum_{i=0}^{\infty}\left(\sum_{j=[t]^{t}+2}^{n+2} M_{j}^{t}\right)^{* i} * V_{t} t^{i} / i !\right\}(x) .
$$

So by Lemma 1 , there exists a nondecreasing function $F^{\prime}$ such that

$$
\left(F_{t, n}\right) \text { c.s. } \stackrel{(c)}{\longrightarrow} F^{\prime}, \text { and T.V. }\left(F^{\prime}\right)=\exp \left(-t \sum_{i=0}^{[t]^{*+1}} \lambda_{i}^{t}\right) \text {. }
$$

But $\sum_{i=0}^{t t^{*}+1} \lambda_{i}^{t}=\lambda_{0}^{t}+\lambda(t)-\lambda(0)=-m^{\prime}(0)+\lambda(t)+m^{\prime}(0)=\lambda(t)$, which implies

$$
\text { T.V. }\left(F^{\prime}\right)=\exp (t \ln m(t) / t)=m(t) .
$$

Claim 8. There exists a sequence of real numbers $\left\{\varepsilon_{n}\right\}$ where $\varepsilon_{n} \downarrow 0$, and there exists a sequence of positive integers $\left\{n_{N}\right\}$ such that if 
1. $\left\{e_{j}: j=[t]+1, \ldots, n\right\}$ is a finite set of positive integers with $\sum_{l=[t]^{*+2}}^{v} e_{l}<j_{v}$ for $n_{N} \leqq v \leqq n$, and

2. $\left\{\tau(l, i):[t]+2 \leqq l \leqq n, n_{N} \leqq i \leqq e_{l}\right\}$ is a collection of positive real numbers such that $\tau(l, i) \in\left[v_{l-1}, v_{l}\right)$, then

for

$$
\underset{l=[t]+2}{\stackrel{n}{*}} \stackrel{e_{l}}{*} G_{i=1} G_{\tau_{l, i}} * V_{t}\left(\bigcup_{k=N ; k \in A(t, \tau)}^{m} A_{k}\right) \geqq\left(1-\varepsilon_{N}\right) V_{t}\left(\bigcup_{k=N ;}^{m} A_{k \in A(t, \tau)}^{m} A_{k}^{\prime}\right)
$$

$$
t \leqq \tau \leqq \tau_{0}=\min \left\{\tau(l, i):[t]+2 \leqq l \leqq n, n_{N} \leqq i \leqq e_{l}\right\}
$$

and $A(t, \tau) \cap\left\{k \in I^{+}: k=N, \ldots, m\right\} \neq \varnothing$.

Proof. Let $\varepsilon_{N}=1 / 2^{N}, n_{N}=N$, and assume $\tau \in\left[t, \tau_{0}\right]$ such that

$$
A(t, \tau) \cap\left\{k \in I^{+}: k=N, \ldots, m\right\} \neq \varnothing .
$$

By 0.1 we see that $*_{l=[t]+2}^{n} *_{i=1}^{e_{l}} G_{\tau(l, i)}$ is the distribution function of a random variable $Y$ of Rubin type with Poissons $\left\{\lambda_{k}^{*}\right\}$ and partition $\left\{b_{n}\right\}$ where $\lambda_{k}^{*}=$ $\sum_{l=[t]^{*}+2}^{n} \sum_{i=1}^{e_{l}} \lambda_{k, \tau(l, i)}$. Now let

$$
\omega \in \bigcap_{n \geqq N ; n \in P}\left(N_{n}(Y)<2^{a_{n}}\right) \cap\left(Y_{t} \in \bigcup_{k=N ;}^{m} A_{k \in A(t, \tau)}^{m}\right) .
$$

So there exists $r \in A(t, \tau)$ with $m \geqq r \geqq N$ such that $Y_{t}(\omega) \in A_{r}^{\prime}$. By reviewing the definition of $A(t, \tau)$ and the definition of the $\lambda_{k, \tau}$ 's we see that $\lambda_{r}^{*}=\lambda_{r+1}^{*}=0$ and therefore

$$
Y(\omega)=\sum_{k=1 ; k \in P}^{r-1}+\sum_{k=r+2 ; k \in P}^{\infty}\left(\sum_{i=0}^{N_{n}(Y)(\omega)} \sum_{j=b_{n}+1}^{b_{n}+1} A_{i, j}(Y)(\omega) 2^{-j}\right) .
$$

Claim 1 implies that the second sum is $\left\langle 2^{-b_{r+1}}\right.$. By the definition of $A_{r}^{\prime}$ we see that (the first sum) $+Y_{t}(\omega) \in A_{r}^{\prime}$.

Therefore $Y(\omega)+Y_{t}(\omega) \in A_{r}$. So since $\left\{N_{n}(Y), Y_{t}: n \in I^{+}\right\}$are an independent collection of random variables we see that

$$
\operatorname{Pr} .\left(Y+Y_{t} \in \bigcup_{k=N ;}^{m} A_{k \in A(t, \tau)} A_{k}\right) \geqq \operatorname{Pr} .\left(\bigcap_{n=N ; n \in P}^{\infty}\left(N_{n}(Y)<2^{a_{n}}\right)\right) \operatorname{Pr} .\left(Y_{t} \in \bigcup_{k=N ;} \bigcup_{k \in A(t, \tau)}^{m} A_{k}^{\prime}\right) .
$$

Now by the hypothesis of this claim

$$
\begin{aligned}
& \lambda_{k}^{*} \leqq 2 j_{l} \ln k \quad \text { if } k \in \bar{P}_{j}, d_{j} \in\left[v_{l-1}, v_{l}\right) \text { and } N \leqq l \leqq n \text {, } \\
& \leqq 2 j_{n} \ln k \quad \text { if } k \in \bar{P}_{j}, d,>n \text {, } \\
& \leqq 2 j_{N} \ln k \text { if } k \in \bar{P}_{j}, d_{j}<N .
\end{aligned}
$$

So for $k \geqq N$, we see that $\lambda_{k}^{*} \leqq \max \left(j_{k}, j(k)\right) \ln k$. Therefore by the definition of the $a_{n}$ 's, and by the fact that the $N_{n}(Y)$ are Poisson random variables we see that

$$
\operatorname{Pr} .\left(\bigcap_{n=N ; n \in P}^{\infty}\left(N_{n}(Y)<2^{a_{n}}\right)\right)>1-1 / 2^{N}
$$

which completes the proof of the claim.

Claim 9. $F^{\prime}\left(\bigcap_{N=1}^{\infty} \bigcup_{k=N ; k \in A(t, \tau)}^{\infty} A_{k}\right)=m(t)$ for $A(t, \tau) \neq \varnothing$.

Proof. Assume $\tau$ is such that $A(t, \tau) \neq \varnothing$. 
Case 1. $t \in D$. By the definition of $A(t, t)$ we see that $A(t, t) \neq \varnothing$. Therefore by Lemma 2, Lemma 8 , and Claim 8 we see that

$$
F^{\prime}\left(\bigcap_{N=1}^{\infty} \bigcup_{k=N ; k \in A(t, t)}^{\infty} A_{k}\right)=m(t) .
$$

But $A(t, \tau) \neq \varnothing$ implies $\tau \geqq t$ and therefore $A(t, \tau) \supset A(t, t)$ which clearly completes the proof of the claim in this case.

Case 2. $t \notin D$. By the definition of $D$ we see that $m$ is continuous from the right at $t$. Let $\left\{t_{i}\right\}$ be a sequence of positive real numbers such that $t_{i} \downarrow t$, and therefore $m\left(t_{i}\right) \rightarrow m(t)$. Since we are assuming $A(t, \tau) \neq \varnothing$ and $t \notin D$ we see that by the definition of $A(t, \tau), \tau>t$. So let $i$ be large enough to insure that $\tau>t_{i}$.

For $n>\left[t_{i}\right]^{*}+1$, let

$$
F_{n}^{t_{l}}=\exp \left(-t \sum_{i=0}^{n+2} \lambda_{i^{t}}^{t}\right)\left\{\sum_{l=0}^{\infty}\left(\sum_{j=\left[t_{i}\right]^{*}+2}^{n+2} M_{j^{t}}^{t^{t}}\right)^{* l} * V_{t} t^{l} / l !\right\} .
$$

By Lemma 1, there exists a nondecreasing function $F^{t_{i}}$ such that $F_{n}^{t_{i}(c)} \stackrel{F^{t_{i}}}{\rightarrow}$. By Lemma 2, Lemma 8 and Claim 8:

$$
F^{t_{i}}\left(\bigcap_{N=1}^{\infty} \bigcup_{k=N ;}^{\infty} A_{k \in A\left(t, t_{i}\right)} A_{k}\right)=\text { T.V. }\left(F^{t_{i}}\right)
$$

But $t_{i}>t$ implies $A(t, \tau) \supset A\left(t, t_{i}\right)$ and therefore

$$
F^{t_{i}}\left(\bigcap_{N=1}^{\infty} \bigcup_{k=N ; k \in A(t, \tau)}^{\infty} A_{k}\right)=\text { T.V. }\left(F^{t_{i}}\right)
$$

Glancing back at the definition of $F^{t_{i}}$ and recalling 0.2 we see that there exists a nondecreasing function $S_{n}(x)$ such that

$$
\left(F_{t, n}\right) \text { c.s. }(x)=S_{n}(x)+F_{n}^{t_{1}}(x) .
$$

But by Lemma $1 F_{n}^{t_{i}} * F_{n, \infty}=F^{t_{i}}$ and therefore $F^{\prime}=S_{n} * F_{n, \infty}+F^{t_{i}}$, which implies

$$
F^{\prime}\left(\bigcap_{N=1}^{\infty} \bigcup_{k=N ;}^{\infty} A_{k \in A(t, \tau)}\right) \geqq F^{t_{i}}\left(\bigcap_{N=1}^{\infty} \bigcup_{k=N ; k \in A(t, \tau)}^{\infty} A_{k}\right)=\text { T.V. }\left(F^{t_{i}}\right) .
$$

Again by Lemma 1

$$
\operatorname{T.V.}\left(F^{t_{i}}\right)=\exp \left(-t \sum_{l=0}^{\left[t_{i}\right]^{*+1}} \lambda_{l^{i}}^{t^{i}}\right)
$$

But

$$
\sum_{i=0}^{\left[t_{i}\right]^{*+1}} \lambda_{l^{i}}^{t}=\lambda_{0}^{t_{i}}+\lambda\left(t_{i}\right)-\lambda(0)=\lambda\left(t_{i}\right)
$$

and therefore

$$
\text { T.V. }\left(F^{t_{i}}\right)=\exp \left(-t \lambda\left(t_{i}\right)\right)
$$


Now since $m$ is continuous from the right at $t$, we see that $\lambda$ is continuous from the right at $t$. So letting $i$ go to $+\infty$, the claim clearly follows.

Let $\tau \in(0, \infty)$ such that $A(t, \tau) \neq \varnothing$. Now by Lemma 3

$$
\left|\bigcap_{n=1}^{\infty} \bigcup_{k=n ; k \in A(t, \tau)}^{\infty} A_{k}\right|=0
$$

and therefore by Claim $9 F^{\prime}$ is continuous singular. From Lemma 1

$$
F_{t}=\left(F_{t, n}\right) \text { a.c. } * F_{n, \infty}+F^{\prime} \text {. }
$$

But clearly the first term is absolutely continuous, and therefore, since $F^{\prime}$ is continuous singular, $\left(F_{t}\right)$ c.s. $=F^{\prime}$. Using the fact that $b_{n} \geqq n(n+1) / 2$, an easy calculation shows that for $j \in I^{+}$,

0.3. $\lim _{n \in \mathcal{P}_{j}}, b_{n} / b_{n+1}=v\left(d_{j}\right)$.

Claim 10. $v(t) \geqq \operatorname{dim} \operatorname{supp}\left(F_{t}\right)$ c.s.

Proof. By Claim 9, Lemma 4, and 0.3 we see that if $A(t, \tau) \neq \varnothing$, then

$$
\operatorname{dim} \operatorname{supp}\left(F_{t}\right) \text { c.s. } \leqq \sup \left\{v\left(d_{j}\right): d_{j} \in A(t, \tau)\right\} \text {. }
$$

If $t \in D$, then $A(t, t) \neq \varnothing$ and by 0.3 Claim 10 is proved. If $t \notin D$, then for each $i \in I^{+}$take $\tau=d_{i}^{\prime}$ and by the definition of $D_{0}$, we see that

$$
\operatorname{dim} \operatorname{supp}\left(F_{t}\right) \text { c.s. } \leqq v(t)
$$

which completes the proof of Claim 10 .

Claim 11. $\operatorname{dim} \operatorname{supp}\left(F_{t}\right)$ c.s. $\geqq v(t)$.

Proof. From Lemma 2.1 if $n \geqq[t]^{*}+1$ then

$$
\left(F_{t}\right) \text { c.s. }=\left(F_{t, n}\right) \text { c.s. } * F_{n, \infty}
$$

and therefore by $\mathrm{A} .3$

By A.5

$$
\operatorname{dim} \operatorname{supp}\left(F_{t}\right) \text { c.s. } \geqq \operatorname{dim} \operatorname{supp}\left(F_{t, n}\right) \text { c.s. }
$$

$$
\operatorname{dim} \operatorname{supp}\left(F_{t, n}\right) \text { c.s. } \geqq \operatorname{dim} \operatorname{supp}\left(\sum_{j=[t]^{*}+2}^{n+2} M_{j}^{t} * V_{t}\right) .
$$

Clearly now by the definition of dim supp we see that in order to prove this claim it is sufficient to show that if $E$ is a Borel set such that

$$
\sum_{j=[t]^{4}+2}^{n+2} M_{j} * V_{t}(E)=\lambda(n)-\lambda(t)
$$

then $\operatorname{dim}(E) \geqq v(t)$. So let $E$ be such a Borel set. Now by Lemma 8 and A.6 we see that $G_{\tau} * V_{t}(E)=1$ for almost all $\tau \in\left[t, v_{n}\right]$ with respect to $\sum_{j=[t]^{*}+2}^{n+2} \lambda_{j}^{t}(\tau)$.

0.4 . Let $\tau_{1}, \tau_{2} \in(0, \infty)$ be such that $\tau \geqq t \geqq \tau_{2}$. If

$$
\sum_{j=1 ;}^{\infty} \sum_{d_{j}<\tau_{2}} 1 / n^{t / d_{j}}<\infty
$$


then by $0.1,0.3$, a review of the definitions of $\lambda_{n, \tau}$ and $\lambda_{n, t}^{\prime}$, and Lemma 5 we see that

$$
\operatorname{dim} \operatorname{supp} V_{t} * G_{\tau} \geqq \inf \left\{v\left(d_{j}\right): d_{j} \in\left[\tau_{2}, \tau\right]\right\}
$$

Case 1. $t \in D$ implies there exists $l \in I^{+}$such that $t=d_{l}$. Let $d_{j}<d_{l}$; then by the way in which we enumerated $D$ we see that:

1. If $j>l$, then $t / d_{j}>c_{j}$ and therefore, by the way $\bar{P}_{j}$ was defined,

$$
\sum_{j=l+1 ; d_{j}<t}^{\infty} \sum_{n \in P_{j}} 1 / n^{t / d_{j}}<\infty
$$

2. If $j<l$, then $t / d_{j}>c_{l}>1$; but there can be only finitely many such $j$ 's and therefore

$$
\sum_{j=1 ; d_{j}<t}^{l-1} \sum_{n \in P_{j}} 1 / n^{t / d_{j}}<\infty
$$

So by 0.4 we see that

$$
\operatorname{dim} \operatorname{supp}\left(G_{\imath} * V_{t}\right) \geqq \inf _{d_{f} \in[t, \tau]} v\left(d_{j}\right),
$$

for all $\tau \in\left[t, v_{n}\right]$ such that $D \cap[t, \tau] \neq \varnothing$.

Subcase i. $m$ is not continuous from the right at $t$. Then, clearly, $\lambda$ is also not continuous from the right at $t$, and therefore $\sum_{j=[t]^{*+2}}^{n+2} \lambda_{j}^{t}(\tau)$ puts positive mass on $\{t\}$. So $G_{t} * V_{t}(E)=1$, which implies $\operatorname{dim}(E) \geqq \operatorname{dim} \operatorname{supp} G_{t} * V_{t}$. But by the above

$$
\operatorname{dim} \operatorname{supp}\left(G_{t} * V_{t}\right) \geqq v(t)
$$

and therefore the claim follows for this subcase.

Subcase ii. $m$ is continuous from the right at $t$. Now by hypothesis if $\varepsilon>0$, then there exists $\delta_{\varepsilon}$ such that $t^{\prime} \in\left(t, t+\delta_{\varepsilon}\right)$ implies

$$
v\left(t^{\prime}\right) \geqq v(t)-\varepsilon .
$$

Also by hypothesis, $\lambda$ is strictly decreasing and therefore $\sum_{j=[t]^{*+2}}^{n+2} \lambda_{j}^{t}(\tau)$ puts positive mass on $\left(t, t+\delta_{\varepsilon}\right)$. So there exists $t^{\prime \prime} \in\left(t, t+\delta_{\varepsilon}\right)$ such that $V_{t} * G_{t^{\prime \prime}}(E)=1$ and therefore $\operatorname{dim}(E) \geqq \operatorname{dim} \operatorname{supp} V_{t} * G_{t^{\prime \prime}}$. But

$$
\operatorname{dim} \operatorname{supp} V_{t} * G_{t^{*}} \geqq \inf \left\{v\left(d_{j}\right): d_{j} \in\left[t, t^{\prime \prime}\right]\right\} \geqq v(t)-\varepsilon
$$

and therefore $\operatorname{dim}(E) \geqq v(t)-\varepsilon$. But $\varepsilon>0$ was arbitrarily selected and therefore the claim follows in this subcase.

Case 2. $t \notin D$. By the definition of $D$ we see that if $\varepsilon>0$, there exists $\delta_{\varepsilon}>0$ such that if $t^{\prime} \in\left(t-\delta_{\varepsilon}, t+\delta_{\varepsilon}\right)$ then $v\left(t^{\prime}\right)>v(t)-\varepsilon$.

By hypothesis $\lambda$ is strictly decreasing and therefore $\sum_{n=[t]^{*+2}}^{n+2} \lambda_{n}^{t}(\tau)$ puts positive 
mass on $\left[t, t+\delta_{\varepsilon}\right)$. So therefore there exists $t^{\prime \prime} \in\left[t, t+\delta_{\varepsilon}\right)$ such that $G_{t^{\prime \prime}} * V_{t}(E)=1$, and so

$$
\operatorname{dim}(E) \geqq \operatorname{dim} \operatorname{supp} G_{t^{n}} * V_{t} .
$$

Choose $\tau^{*} \in\left(t-\delta_{\varepsilon}, t\right)$; then $t / d_{j}>t / \tau^{*}>1$ for all $d_{j}<\tau^{*}$ and therefore

$$
\sum_{d_{j}<\tau^{*}} \sum_{n \in \mathbb{P}_{j}} 1 / n^{t / d_{j}}<\infty
$$

So by 0.4

$$
\operatorname{dim} \operatorname{supp} V_{t} * G_{t^{\prime \prime}} \geqq \inf \left\{v\left(d_{j}\right): d_{j} \in\left[\tau^{*}, t^{\prime \prime}\right]\right\} \geqq v(t)-\varepsilon
$$

and therefore $\operatorname{dim}(E) \geqq v(t)-\varepsilon$. But $\varepsilon$ was just some number greater than zero, and therefore the claim is established in this case, exhausting all possibilities.

Our theorem is now established if $m(t) \neq 0$ or $m^{\prime}(0)$ for $t>0$. To prove this theorem in general just repeat the above arguments with the following modifications.

Let

$$
\alpha=\text { l.u.b. } A \quad \beta=\text { g.l.b. } B \text {. }
$$

Now let $\left\{v_{n}\right\}$ be a sequence of positive real numbers such that $v_{n} \geqq \alpha$ and $v_{n} \uparrow \beta$. For notational convenience we let $v_{0}=\alpha$. Now define $D_{1}, D_{2}$ and $D_{3}$ as before but now restrict them to $(\alpha, \beta)$. Now let

$$
D_{4}=\{t \in A: v \text { is not continuous at } t\} \text {. }
$$

Since by hypothesis $v$ is monotone on $D_{4}$, we see that $D_{4}$ is countable. Now we modify $D$ to be

$$
D=\bigcup_{i=1}^{4} D_{i} \cup\{\alpha, \beta\}
$$

Again $D$ is countable and we enumerate $D$ as before with the restriction that $c_{n}<1+\beta / n$.

The only other modifications we need make is that in the definition of $M_{n}^{t}$ we restrict $t \in(\alpha, \beta)$ and

$$
M_{0}^{t}(x)=\left\{-m^{\prime}(0) V(x)+\lambda^{\alpha} G^{\alpha}(x)+\lambda^{\beta} U(x)-\left(\lambda(\alpha)+\lambda^{\beta}\right)\right\}
$$

where $\lambda^{\alpha}=m^{\prime}(0)+\lambda(\alpha)$,

$$
\begin{aligned}
\lambda^{\beta} & =\lambda(\beta)-\lambda(\beta-0) & & \text { if } m(\beta) \neq 0, \\
& =0 & & \text { if } m(\beta)=0 .
\end{aligned}
$$

$U(x)$ is the distribution function whose induced measure puts mass one on $\{0\}$, and $G^{\alpha}$ is the distribution function of a Rubin type random variable with Poissons $\left\{\lambda_{n}^{\alpha}\right\}$ and partition $\left\{b_{n}\right\}$ where

$$
\begin{aligned}
\lambda_{n}^{\alpha} & =2 \ln n & & \text { if } n \in \bar{P}_{j}, d_{j} \leqq \alpha, \\
& =0 & & \text { otherwise. }
\end{aligned}
$$


Corollary. Let $\alpha \in(0,+\infty]$. If $v(t)$ maps $(0, \alpha)$ into $[0,1]($ or $(0, \alpha]$ if $\alpha<+\infty)$ such that $v(t) \leqq \lim \inf _{\tau \rightarrow t^{+}} v(\tau)$ at all but possibly a countable number of points of $(0, \alpha)$, then there exists a stochastic process, $\left\{X_{t}: t \in[0, \infty)\right\}$ with stationary independent increments such that

(1) if $t \in(0, \alpha)$ (or $t \in(0, \alpha])$, then $\operatorname{dim} \operatorname{supp}\left(F_{t}\right)$ c.s. $=v(t)$, and

(2) if $t \in[\alpha, \infty)($ or $t \in(\alpha, \infty))$, then $F_{t}$ is absolutely continuous.

Proof. We prove the corollary for $(0, \alpha)$; then a clear modification of this proof will establish this corollary for $(0, \alpha]$. Let $X=\left\{x \in(0, \alpha): v(x)>\lim \inf _{\tau \rightarrow x^{+}} v(x)\right\}$ be countable and let $\left\{x_{i}\right\}$ be an enumeration of $X$. Let $\left\{\xi_{i}\right\}$ be a sequence of real numbers such that $\sum \xi_{i}<\infty$. Finally for $t \in(0, \alpha)$ let

$$
\lambda(t)=\left(1+\sum_{d_{i}<t} \xi_{i}\right) t
$$

So for $t \in(0, \infty)$, let

$$
\begin{aligned}
m(t) & =\exp -t \lambda(t) & & \text { if } t \in(0, \alpha), \\
& =0 & & \text { if } t \notin(0, \alpha) .
\end{aligned}
$$

Let $v^{\prime}(t)$ be defined as

$$
\begin{aligned}
v^{\prime}(t) & =v(t) & & \text { if } t \in(0, \alpha), \\
& =0 & & \text { if } t \notin(0, \alpha) .
\end{aligned}
$$

Now $v^{\prime}(t)$ and $m(t)$ clearly satisfy the hypothesis of our theorem and the corollary follows.

All the conditions imposed on $m(t)$ and $v(t)$, with the exception that $\ln (m(t)) / t$ be strictly decreasing on $(0, \infty)-(A \cup B)$ are certainly necessary conditions in order that there exists a stochastic process, $\left\{X_{t}, t \in[0, \infty)\right\}$, with stationary independent increments such that T.V. $\left(F_{t}\right)$ c.s. $=m(t)$, and $v(t)=\operatorname{dim} \operatorname{supp}\left(F_{t}\right)$ c.s.

The author has been unable to determine what happens if $\ln m(t) / t$ is not strictly decreasing on $(0, \infty)-(A \cup B)$. In fact, the author has not even been successful in finding a process $\left\{X_{t}: t \in[0, \infty)\right\}$ with continuous distribution functions and with stationary independent increments such that $\ln \left(\right.$ T.V. $\left(F_{t}\right)$ c.s. $) / t$ increases over some interval $(\alpha, \beta)$ where $\alpha, \beta \in(0, \infty)$.

\section{REFERENCES}

1. A. M. Bruckner and E. Ostrow, Some function classes related to the class of convex functions, Pacific J. Math. 12 (1962), 1203-1215.

2. S. D. Chatterji, Certain induced measures and the fractional dimension of their suprorss, Z. Wahrscheinlichkeitstheorie und Verw. Gebiete 3 (1964), 184-193.

3. B. V. Gnedenko and A. N. Kolmogorov, Limit distributions for sums of independent random variables, Addison-Wesley, Cambridge, Mass., 1954. 
4. P. Hartman and A. Wintner, On the infinitesimal generators of integral convolutions, Amer. J. Math. 64 (1942), 273-298.

5. H. Rubin, Supports of infinitely divisible distributions, Tech. Rep. No. RM-108, HR-10, Michigan State Univ., Dept. of Statistics, East Lansing, Mich., 1963.

6. Stanislaw Saks, Theory of the integral, Dover, New York, 1964.

7. H. G. Tucker, On a necessary and sufficient condition that an infinitely divisible distribution be absolutely continuous, Trans. Amer. Math. Soc. 118 (1965), 316-330.

UNiVersity OF CALIForNia,

Riverside, California 